\title{
Ultrafast ytterbium-doped bulk lasers and laser amplifiers
}

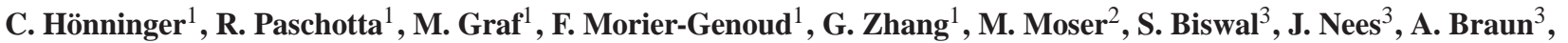 \\ G.A. Mourou ${ }^{3}$, I. Johannsen ${ }^{4}$, A. Giesen ${ }^{4}$, W. Seeber ${ }^{5}$, U. Keller ${ }^{1}$ \\ ${ }^{1}$ Ultrafast Laser Physics, Institute of Quantum Electronics, Swiss Federal Institute of Technology, ETH Hönggerberg - HPT, CH-8093 Zürich, Switzerland \\ (Fax: +41-1/633-1059, E-mail: paschotta@iqe.phys.ethz.ch) \\ ${ }^{2}$ CSEM Zürich, Badenerstrasse 569, CH-8048 Zürich, Switzerland \\ ${ }^{3}$ Center of Ultrafast Optical Science, University of Michigan, Ann Arbor, MI 48108, USA \\ ${ }^{4}$ Institut für Strahlwerkzeuge, Universität Stuttgart, Pfaffenwaldring 43, D-70569 Stuttgart, Germany \\ ${ }^{5}$ Otto-Schott-Institut, Universität Jena, Fraunhoferstr. 6, D-07743 Jena, Germany
}

Received: 4 January 1999/Revised version: 15 March 1999/Published online: 26 May 1999

\begin{abstract}
We review recent achievements with ultrafast bulk lasers and amplifiers based on Yb:YAG and Yb:glass. Special attention is paid to those aspects of the quasi-three-level nature of these gain media that are important for short-pulse generation. The potential of Yb-doped media for efficient short-pulse generation and amplification is compared to their Nd-doped counterparts.
\end{abstract}

PACS: $42.60 ; 42.55$

In recent years, the $\mathrm{Yb}^{3+}$ ion has been recognized as an interesting dopant for solid-state laser materials $[1,2]$. The $\mathrm{Yb}^{3+}$ ion has a very simple energy level scheme, which consists of only two manifolds participating in the lasing process. The laser operation wavelength is in the $1 \mu \mathrm{m}$ region, similar to the $\mathrm{Nd}^{3+}$ ion.

Yb-doped laser materials can be pumped by strained layer InGaAs diode lasers in the wavelength range between $0.9 \mu \mathrm{m}$ and $1.1 \mu \mathrm{m}$. Very efficient lasing is possible because of the small quantum defect (for example about 3 times less than for $\mathrm{Nd}$ on the $1.06 \mu \mathrm{m}$ transition [3]). In addition, the efficiency is not degraded by processes such as concentration quenching and excited-state absorption. In contrast to many other solidstate laser materials, high doping levels are possible without a reduction of the upper-state lifetime. Short pump absorption lengths can thus be obtained, somewhat lowering the (relatively high) demands on the beam quality of the laser diodes used for pumping, and also minimizing material dispersion and Kerr nonlinearity. Problems originating from the thermal load, which in high-power lasers can be severe at high doping levels, are minimized by the high intrinsic efficiency.

Although the small quantum defect results in good efficiency, it causes a significant thermal population even in the highest Stark levels of the laser manifolds. As the higher Stark levels of the ground state manifold are used as lower laser levels, this population leads to quasi-three-level characteristics with ground-state absorption at the laser wavelength and thus significantly increased laser threshold pump intensities. By cooling of the gain medium, the lower laser level population can substantially be reduced. This decreases the laser threshold and thus improves the laser efficiency.

Stimulated emission of the $\mathrm{Yb}^{3+}$ ion was first observed in an oxide glass [4] under cryogenic conditions. Yb:YAG was investigated in the early 1970s. Reinberg et al. used Si:GaAs light-emitting diodes as pump sources [5]. In this experiment both the $\mathrm{Yb}$ :YAG crystal and the pump diodes required cryogenic operation. From that point it took 20 years until the development of efficient InGaAs diode lasers resulted in the first room-temperature diodepumped Yb:YAG laser [6]. Enormous progress has been achieved since then. Excellent performance of diode-pumped Yb:YAG lasers with continuous-wave (cw) output powers as high as $346 \mathrm{~W}$, up to $50 \%$ optical efficiency, and almost diffraction-limited output beam quality have been demonstrated recently using the so-called thin-disc concept $[7,8]$. Due to the higher emission cross section compared to Yb:YAG, the Yb-doped S-FAP crystal became interesting as a laser material in recent years $[9,10]$. Additionally, the cost-effectiveness of glass as host material for solid-state lasers led to the development and investigation of various Yb-doped glass bulk lasers [1114]. Yb-doped glasses are also used in fiber form for lasers [15, 16] and amplifiers [17]. Recently, $35 \mathrm{~W}$ cw output power was demonstrated from a single-mode $\mathrm{Yb}$-doped fiber laser [18].

In this paper we discuss the advantages of $\mathrm{Yb}$-doped laser materials for the generation of short pulses and high peak powers, as required for numerous applications. In comparison to the corresponding Nd-doped laser materials, Yb-doped media show substantially broader emission spectra, which allow for shorter pulse generation and wider wavelength tuning. Pulse durations of $60 \mathrm{fs}$ and a tuning range from 1.03 to $1.08 \mu \mathrm{m}$ have been obtained from Yb:glass bulk lasers [14] and $65 \mathrm{fs}$ from an Yb-doped fiber laser [19]. Sub-picosecond pulses can be obtained even from crystalline Yb-doped materials such as Yb:YAG [20], whereas the $\mathrm{Nd}^{3+}$ ion provides 
sufficient emission bandwidth for sub-picosecond pulses only with glasses as host materials $[21,22]$. The much better thermal properties of $\mathrm{Yb}$-doped crystals compared to $\mathrm{Nd}$-doped glasses are very favorable especially for high-power ultrafast applications.

We use semiconductor saturable absorber mirrors (SESAMs) [23,24] for passive modelocking. The SESAM parameters can be custom-designed to obtain the shortest pulse duration and stable cw modelocking without Qswitching instabilities [25-27], despite the small cross sections of Yb-doped laser media. We achieved the shortest pulses from Yb:YAG lasers (340 fs) as well as for Yb:glass lasers (60 fs) (see Sects. 2.1 and 2.2, respectively). Other groups have demonstrated 160-fs pulses from a Kerr lens modelocked Yb:glass laser, pumped with a Ti:sapphire laser [28]. Active modelocking leads to longer pulses of $80 \mathrm{ps}$ for Yb:YAG [29] and 35 ps for Yb:CS-FAP [30].

In Sect. 3, we discuss the advantages of Yb-doped laser media in regenerative amplifiers for energetic ultrashort pulses. Recent experiments have shown that the large energy storage capability and particularly the broad amplification bandwidth (compared to Nd-doped media) are advantageous. For example, we obtained 2.3-ps short pulses from an Yb:YAG regenerative amplifier [31]. This is an order of magnitude shorter than that obtained with Nd:YAG [32]. Compact laser amplifiers, which provide ultrashort laser pulses at the microjoule to millijoule energy level, are of interest for applications such as medical surgery [33] of micro-machining [34].

\section{Yb:YAG and Yb:glass as quasi-three-level laser materials}

Quasi-three-level systems are characterized by a thermal population in the lower laser level, which leads to re-absorption at the laser wavelength and causes high laser thresholds. To fully exploit the potential for highly efficient operation of
Yb-doped lasers, high-brightness laser diodes are required as pump sources because they can be focused to small spots while maintaining moderate beam divergence. High-power, low-brightness diode bar pump sources result in large mode areas in the gain medium and thus in high laser thresholds and relatively low efficiency. Extensive numerical investigations of longitudinally pumped quasi-three-level lasers can be found in [35-37]. In the following we briefly address the most important consequences by applying local rate equations.

\subsection{Effective gain profile}

The gain coefficient $g$, related to the power gain factor $G$ by $G=\exp (g)$, is given by

$$
\begin{aligned}
g & \equiv 2^{*} L_{\mathrm{g}}\left(N_{2} \sigma_{\mathrm{em}}^{(L)}-N_{1} \sigma_{\mathrm{abs}}^{(L)}\right) \\
& =2^{*} L_{\mathrm{g}}\left[N_{2}\left(\sigma_{\mathrm{em}}^{(L)}+\sigma_{\mathrm{abs}}^{(L)}\right)-N_{\mathrm{tot}} \sigma_{\mathrm{abs}}^{(L)}\right],
\end{aligned}
$$

where $\sigma_{\mathrm{em}}^{(L)}$ and $\sigma_{\mathrm{abs}}^{(L)}$ are the effective emission and absorption cross sections of the laser transition, $N_{1}$ and $N_{2}$ are the population densities in the lower and upper laser manifold, $L_{\mathrm{g}}$ is the length of the gain medium, and the factor $2^{*}$ symbolizes the number of passes through the gain medium per cavity round trip. It is 2 for a standing wave cavity and 1 for a ring cavity. Here, $N_{\text {tot }}=N_{1}+N_{2}$ is the total number of active ions per unit volume.

Figure 1 shows the measured effective emission and absorption cross sections of Yb-doped YAG (Fig. 1a) and of a Yb-doped glass (Kigre QX/Yb phosphate, Fig. 1b). The absolute scaling of the emission cross sections was determined by the assumption that absorption and emission cross sections are identical at the peaks of $968 \mathrm{~nm}$ and $975 \mathrm{~nm}$, respectively. For comparison we list the important laser parameters of Yb:YAG and Nd:YAG [38] in Table 1.

Table 1. $Y b: Y_{A G}{ }^{a)}$ and $N d: Y A G^{b)}$ parameters

\begin{tabular}{lccc}
\hline Laser material & \multicolumn{2}{c}{ Yb:YAG } & Nd:YAG \\
\hline Laser wavelength $/ \mu \mathrm{m}$ & 1.03 & 1.05 & 1.064 \\
Emission bandwidth $\Delta \lambda / \mathrm{nm}$ & 6.3 & 5.6 & 0.6 \\
Emission cross section $\sigma_{\mathrm{em}}^{(L)} / \times 10^{-20} \mathrm{~cm}^{2}$ & 2.1 & 0.31 & 33 \\
Absorption cross section $\sigma_{\text {abs }}^{(L)} / \times 10^{-20} \mathrm{~cm}^{2}$ & 0.1 & 0.01 & 0 \\
Gain saturation fluence ${ }^{\mathrm{c})} F_{\mathrm{sat}, \mathrm{L}} / \mathrm{J} / \mathrm{cm}^{2}$ & 8.8 & 30 & 0.57 \\
Fluorescence lifetime $\tau / \mathrm{ms}$ & 0.95 & 0.95 & 0.23 \\
Absorption cross section ${ }^{\mathrm{d})} \sigma_{\text {abs }}^{(P)} / \times 10^{-20} \mathrm{~cm}^{2}$ & 0.75 & 0.75 & 8.6 \\
Emmision cross section ${ }^{\mathrm{d})} \sigma_{\mathrm{em}}^{(P)} / \times 10^{-20} \mathrm{~cm}^{2}$ & 0.07 & 0.07 & - \\
Pump saturation intensity ${ }^{\mathrm{d})} I_{\mathrm{sat}, \mathrm{P}} / \mathrm{kW}^{2} / \mathrm{cm}^{2}$ & 27 & 27 & 9 \\
Transparency intensity $I_{\mathrm{P}, \mathrm{trans}}^{(L)} / \mathrm{kW} / \mathrm{cm}^{2}$ & 1.4 & 1.0 & 0 \\
Doping density $N_{\text {tot }}$ for $1 \% \mathrm{R}^{3+} / \times 10^{20} \mathrm{~cm}^{-3}$ & 1.38 & 1.38 & 1.38 \\
Thermal conductivity $/ \mathrm{W} \mathrm{cm}^{-1} \mathrm{~K}^{-1}$ & & 0.14 & \\
Refractive index & & 1.82 & \\
Nonlinear refractive index $/ \times 10^{-16} \mathrm{~cm}^{2} / \mathrm{W}$ & & 6.9 & \\
\hline
\end{tabular}

a) data from our measurements (top 10 lines) and from [38] (last 4 lines),

b) data taken from [38],

c) note that the factor of $2^{*}$ is not taken into account here,

d) data for Yb:YAG at $0.94 \mu \mathrm{m}$ and for Nd:YAG at $0.809 \mu \mathrm{m}$ wavelength 


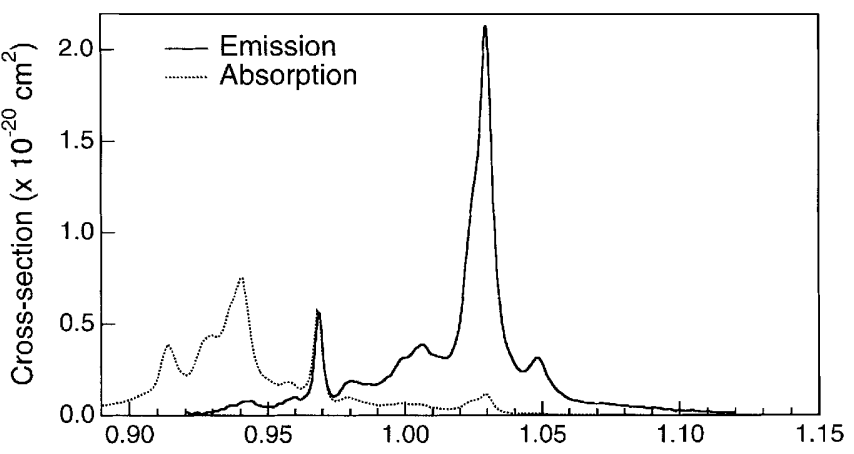

a) Wavelength $(\mu \mathrm{m})$



Fig. 1a,b. Measured absorption (dotted) and emission (solid) cross sections of a a 5 at. \% doped $\mathrm{Yb}$ :YAG crystal and for $\mathbf{b}$ a Kigre QX phosphate glass doped with $15 \% \quad \mathrm{Yb}_{2} \mathrm{O}_{3}$

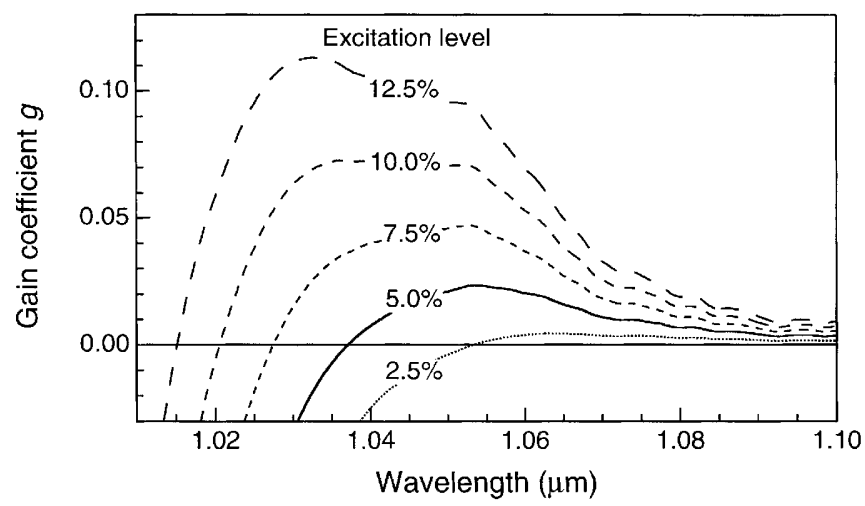

Fig. 2. The dependence of the effective spectral gain curve on the cavity loss (excitation level) is shown for a QX/Yb phosphate glass laser. Because of the lower influence of re-absorption at higher inversion levels, the peak of the gain spectrum shifts to shorter wavelengths. Additionally, the effective gain spectrum broadens. See also Table 2

At steady state, the laser gain just compensates for the cavity loss. This means that the excitation level, i.e. the fraction of laser ions in the upper manifold, is clamped at a fixed value determined by the cavity loss and the length and the doping level of the gain medium (see (1)). In Fig. 2, we illustrate the gain spectrum for various excitation levels of the Yb-doped phosphate glass calculated from the data in Fig. 1b. We assumed $7 \mathrm{~mm}$ round-trip gain length and $N_{\text {tot }}=2 \times 10^{21} \mathrm{~cm}^{-3}$, which corresponds to $\approx 15 \% \mathrm{Yb}_{2} \mathrm{O}_{3}$ doping concentration. In contrast to four-level lasers, the peak wavelength and the gain bandwidth depend on the excitation level and thus on the doping level and on the cavity loss (see Table 2). For Yb:YAG, the situation is different due to
Table 2. Saturated gain of QX/Yb phosphate glass laser

\begin{tabular}{cccc}
\hline $\begin{array}{c}\text { Excitation level, } \\
N_{2} / N_{\text {tot }} / \%\end{array}$ & $\begin{array}{c}\text { Cavity loss } \\
/ \%\end{array}$ & $\begin{array}{c}\text { Peak wavelength } \\
/ \mu \mathrm{m}\end{array}$ & $\begin{array}{c}\text { FWHM gain } \\
\text { bandwidth } / \mathrm{nm}\end{array}$ \\
\hline 2.5 & 0.4 & 1.063 & 26.3 \\
5 & 2 & 1.054 & 26.8 \\
7.5 & 4 & 1.053 & 35.3 \\
10 & 5.7 & 1.044 & 41.0 \\
12.5 & 7.5 & 1.033 & 43.8 \\
\hline
\end{tabular}

the pronounced $1030-\mathrm{nm}$ and 1050 -nm peaks in the emission spectrum; here a change of the cavity loss and thus the inversion level could induce a sudden jump between a laser wavelength near $1030 \mathrm{~nm}$ and another one near $1050 \mathrm{~nm}$, while the changes of emission wavelength are small as long as such a jump does not occur.

\subsection{Optimization of crystal length}

In an end-pumped quasi-three-level laser, optimum power efficiency is achieved when the residual pump intensity at the end of the gain medium is just sufficient to maintain transparency (i.e. zero loss) at this point. Assuming a top-hat transverse profile of the pump and laser beam, this leads to the condition

$I_{\mathrm{P}, \text { out }} \equiv I_{\mathrm{P}, \text { trans }}^{(L)}=\frac{h \nu_{\mathrm{P}}}{\left(\sigma_{\mathrm{abs}}^{(P)} \sigma_{\mathrm{em}}^{(L)} / \sigma_{\mathrm{abs}}^{(L)}-\sigma_{\mathrm{em}}^{(P)}\right) \tau}$.

From this relation we see that the pump absorption efficiency $\eta_{\mathrm{abs}}$ is limited by the available pump intensity $I_{\mathrm{P} \text {,in }}$ to $\eta_{\mathrm{abs}}=1-I_{\mathrm{P} \text {,trans }} / I_{\mathrm{P} \text {,in }}$, assuming constant beam area and a single pass of the pump light. For pumping with high-power low-brightness diode lasers, the efficiency can be seriously degraded by the relatively low available pump intensity.

For fs lasers based on Yb:glass, these considerations also influence the center wavelength and achievable pulse duration, as shown in the following. When the length and doping level of the gain medium are chosen for optimum power efficiency, the inversion level in the gain medium will still depend on the cavity loss. With low output coupler transmission, the inversion level will be low as well, resulting in operation at relatively long wavelengths and small amplification bandwidth, as discussed above. For higher output coupler transmission, a shorter center wavelength and broader FWHM (full width at half maximum) gain bandwidth is achieved. However, the limiting effect of the gain filter depends not only on the FWHM gain bandwidth $\Delta \lambda_{\mathrm{g}}$ but also on the peak gain coefficient $g_{\text {peak. }}$. It can be shown $[39,40]$ that the minimum achievable pulse duration is approximately proportional to $\left(g_{\text {peak }}\right)^{1 / 2} / \Delta \lambda_{\mathrm{g}}$. In effect, the minimum pulse duration of an Yb:glass laser is achieved with weak output coupling, i.e. low inversion (a few percent), despite the smaller value of $\Delta \lambda_{\mathrm{g}}$.

Still shorter pulses would require both a high inversion level and a small cavity loss. This inevitably means that a shorter crystal or lower doping level must be used, thus compromising the pump absorption efficiency. We expect that, in this way, the pulse duration could be reduced somewhat below the record value of $60 \mathrm{fs}$ demonstrated so far [14]. 
The shorter center wavelength would then also be more suitable for seeding a regenerative amplifier, which is typically operated at higher average inversion levels.

\subsection{Passive modelocking using SESAMs}

For passive modelocking we use an intracavity semiconductor saturable absorber mirror (SESAM). A detailed description of this type of saturable absorber can be found elsewhere $[23,24]$. Here, we only summarize the most important parameters and their influence on the dynamics of passively modelocked lasers.

We distinguish between two basic SESAM devices, namely high-finesse [41,42] and low-finesse [20,43-45] antiresonant Fabry-Pérot saturable absorbers (A-FPSAs). An A-FPSA consists of a semiconductor saturable absorber structure, which is embedded between two mirrors. One mirror, which we call the bottom mirror, has a high reflectivity of $>99 \%$ in all A-FPSA designs and consists of 16 to 25 pairs of AlAs/GaAs quarter-wave layers. The second Fabry-Pérot mirror (top reflector) can have different reflectivity and determines whether we call a design high-finesse or low-finesse.

Saturable absorbers for ultrashort pulse generation must be broadband devices with low group delay dispersion (GDD). This is achieved by operating the Fabry-Pérot saturable absorber at antiresonance. For antiresonance, the optical thickness of the absorber structure must be adjusted such that the round trip phase $\Phi_{\mathrm{rt}}$ in the Fabry-Pérot produces destructive interference (for example $\Phi_{\mathrm{rt}}=(2 m-1) \pi$, $m=1,2, \ldots)[42]$.

High-finesse A-FPSAs have a high-reflecting top mirror (for example 95\%) which is evaporated on the semiconductor absorber after the molecular beam epitaxial growth. The high reflectivity of the top mirror results in a large absorber saturation fluence $F_{\text {sat,A }}$ and additionally requires a thick absorber $(\approx 0.61 \mu \mathrm{m})$ to achieve a significant modulation depth $\Delta R$. Here, $F_{\text {sat, }}$ is the pulse fluence on the SESAM necessary to bleach the saturable absorption to 1 /e of its maximum amount $\Delta R$.

Low-finesse A-FPSAs avoid any post-growth processing since the top mirror is formed by the air-GaAs interface, which leads to $\approx 30 \%$ Fresnel reflection. The lower reflectivity of the top mirror substantially reduces the absorber saturation fluence. Besides that, a significant modulation depth is obtained with a thin absorber layer of 20 to $50 \mathrm{~nm}$ thickness. An additional reduction of the saturation fluence can be obtained by placing the absorber layer close to the antinode

Table 3. SESAM parameters

\begin{tabular}{cccccc}
\hline $\begin{array}{c}\text { SESAM } \\
\#\end{array}$ & Structure & $\begin{array}{c}\text { Absorber } \\
\text { thickness } \\
/ \mathrm{nm}\end{array}$ & $\begin{array}{c}\text { Modulation } \\
\text { depth, } \Delta R \\
/ \%\end{array}$ & $\begin{array}{c}\text { Saturation } \\
\text { fluence, } F_{\text {sat, } \mathrm{A}} \\
/ \mu \mathrm{J} / \mathrm{cm}^{2}\end{array}$ & $\begin{array}{c}\text { Recovery } \\
\text { time, } \tau_{\mathrm{A}} \\
/ \mathrm{ps}\end{array}$ \\
\hline & & & & & \\
1 & High-finesse & $610(\mathrm{MQW})$ & $\approx 0.25$ & $\approx 4500$ & 4 \\
2 & Low-finesse & 30 & $\approx 0.5$ & $\approx 120$ & 6 \\
3 & Low-finesse & 25 & $\approx 0.7$ & $\approx 100$ & 6 \\
4 & Low-finesse & 20 & $\approx 0.5$ & $\approx 100$ & 6 \\
5 & Low-finesse & 50 & $\approx 1.3$ & $\approx 120$ & 6 \\
6 & Low-finesse & 25 & $\approx 0.9$ & $\approx 120$ & 5 \\
7 & Low-finesse & 15 & $\approx 0.7$ & $\approx 120$ & 15 \\
\hline
\end{tabular}

of the standing-wave intensity distribution inside the FabryPérot structure. The thick absorber layer of the high-finesse A-FPSA covers several oscillations of the electromagnetic field distribution, effectively eliminating such standing-wave effects.

Another important macroscopic SESAM parameter is the recovery time $\tau_{\mathrm{A}}$, which can be controlled mainly with the growth temperature and is measured in a pump-probe experiment. The parameters of the SESAMs used in our experiments are listed in Table 3.

The applied passive modelocking mechanism depends on the pulse duration regime. In ps lasers, operating with some uncompensated positive dispersion, the SESAM usually acts as a fast saturable absorber $[39,46]$. The fs lasers (and some of the ps lasers) with intracavity prisms for dispersion compensation are soliton modelocked $[47,48]$. Here, the pulse shaping is dominated by the interplay of the negative cavity group delay dispersion (GDD) and self phase modulation (SPM), not by the SESAM which typically acts as a slow absorber and only starts and stabilizes the soliton pulses. Pulse widths well below the SESAM response time are routinely obtained.

\section{$1.4 Q$-switched modelocking}

The use of a saturable absorber as a passive modelocker in a solid-state laser introduces a tendency for Q-switched modelocking (QML) operation [25-27]. In the QML operation regime, the laser output consists of modelocked pulses underneath a Q-switched envelope, i.e., the laser power exhibits undesirably large amplitude fluctuations on the $\mathrm{kHz}$ frequency level. In [27], we investigated this issue in detail for ps as well as for soliton-modelocked fs four-level lasers. Here, we briefly summarize the results and address the situation of quasi-three-level lasers.

To achieve continuous-wave (cw) modelocking without Q-switching instabilities, the intracavity pulse energy $E_{\mathrm{P}}$ must exceed a certain value, the critical pulse energy $E_{\mathrm{P}, \mathrm{c}}$. For ps lasers, we have derived the following expression for the critical pulse energy [27]:

$E_{\mathrm{P}, \mathrm{c}}=\left(E_{\mathrm{sat}, \mathrm{L}} E_{\mathrm{sat}, \mathrm{A}} \Delta R\right)^{1 / 2}=\left(F_{\mathrm{sat}, \mathrm{L}} A_{\mathrm{eff}, \mathrm{L}} F_{\mathrm{sat}, \mathrm{A}} A_{\mathrm{eff}, \mathrm{A}} \Delta R\right)^{1 / 2}$

Here, $F_{\text {sat,L }}$ is the gain saturation fluence and $A_{\text {eff,L }}$ and $A_{\text {eff,A }}$ denote the effective laser mode areas in the gain medium and on the SESAM, respectively. We refer to the term on the righthand-side as the "QML parameter". A small QML parameter is required to achieve stable modelocking at moderate intracavity pulse energy. Relation (3), although originally derived for four-level lasers, is valid for quasi-three-level lasers as well. We only have to calculate the different gain saturation behavior from

$F_{\mathrm{sat}, \mathrm{L}}=h v_{\mathrm{L}} /\left[2^{*}\left(\sigma_{\mathrm{em}}^{(L)}+\sigma_{\mathrm{abs}}^{(L)}\right)\right]$,

where $h v_{\mathrm{L}}$ is the laser photon energy. Relation (4) can be derived from the quasi-three-level laser rate equations.

Short-pulse lasers require gain media with broad emission bandwidth. These typically have significantly smaller 
cross sections (with the exception of Ti:sapphire). In particular, Yb-doped media have significantly broader emission bandwidths than their Nd-doped counterparts, but also have about an order of magnitude lower cross sections. The result is a high gain saturation energy and, thus, a strong tendency for QML, particularly for Yb-doped glasses. However, we found experimentally and by theoretical modeling [27] that in soliton modelocked lasers (with negative intracavity GDD) the critical pulse energy is typically a factor of $\approx 4$ lower than calculated with (3). This is due to the interplay of soliton effects and the gain filter.

We managed to suppress QML in passively modelocked Yb-doped lasers by minimizing the pump spot size, focusing more tightly on the SESAM and limiting the SESAM's modulation depth. Yb:YAG, with its higher cross sections (compared to Yb:glass), allowed for the suppression of QML even when pumping with high-power low-brightness diodes (larger pump spot). Here, the stabilizing effect of soliton modelocking in a dispersion-compensated cavity (as mentioned above) was employed. This also led to shorter pulses and higher peak powers.

\subsection{Selection of laser wavelength}

The Yb:YAG emission spectrum (see Fig. 1a) allows for operation near $1.03 \mu \mathrm{m}$ or $1.05 \mu \mathrm{m}$. In the following, we show how the actual wavelength of operation depends on the cavity parameters and how the wavelength selection can be influenced by using a specially designed SESAM.

For low cavity loss, $1.05 \mu \mathrm{m}$ operation is favored by the quasi-three-level nature (strong re-absorption at $1.03 \mu \mathrm{m}$ ). This tendency is often increased by SESAMs: for shorter wavelengths, the absorber action is stronger because of the increased density of states in the semiconductor saturable

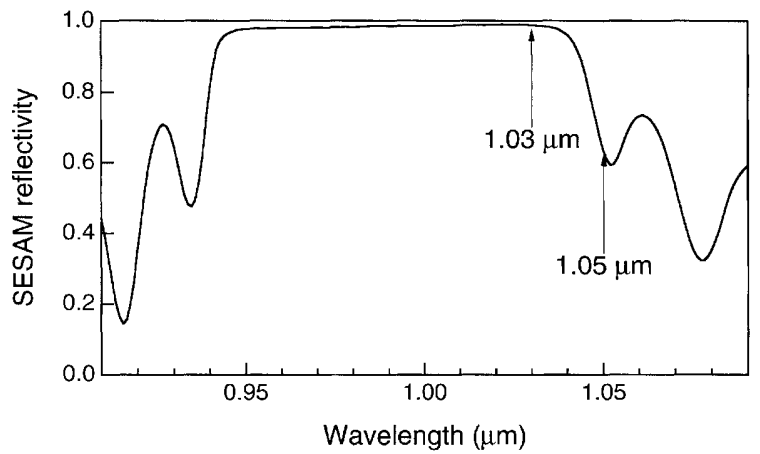

Fig. 3. Reflectivity curve of a SESAM (\#3 of Table 3), which is designed for operation at $1.03 \mu \mathrm{m}$ wavelength by shifting the bottom mirror reflectivity to shorter wavelengths. The GaAs and AlAs layer thicknesses of the bottom mirror were $71 \mathrm{~nm}$ and $84 \mathrm{~nm}$, respectively (corresponds to a center wavelength of $1.00 \mu \mathrm{m}$ ). For common bottom mirrors, which are centered at $1.05 \mu \mathrm{m}$, the layer thicknesses are $75 \mathrm{~nm}$ for GaAs and $89 \mathrm{~nm}$ for AlAs

absorber. It can be desirable to obtain laser operation at $1.03 \mu \mathrm{m}$ because the slightly broader emission spectrum enables the generation of shorter modelocked pulses or because the higher cross sections help to suppress QML. There are two ways in which SESAMs can be used to select this shorter wavelength.

The first way is operation near the long wavelength edge of the highly reflecting range of the SESAM bottom mirror. For example, we can design the bottom mirror with a center wavelength at $0.99 \mu \mathrm{m}$, which results in high reflectivity at $1.03 \mu \mathrm{m}$ and significantly lower reflectivity at $1.05 \mu \mathrm{m}$ (Fig. 3, SESAM \#3 in Table 3). We can also use such a SESAM to shift the center wavelength of a $\mathrm{Yb}$ :glass laser to shorter wavelengths at low inversion levels, where the peak gain is at longer wavelengths (see Sect. 1.1). The limitation of this approach is that operation a)
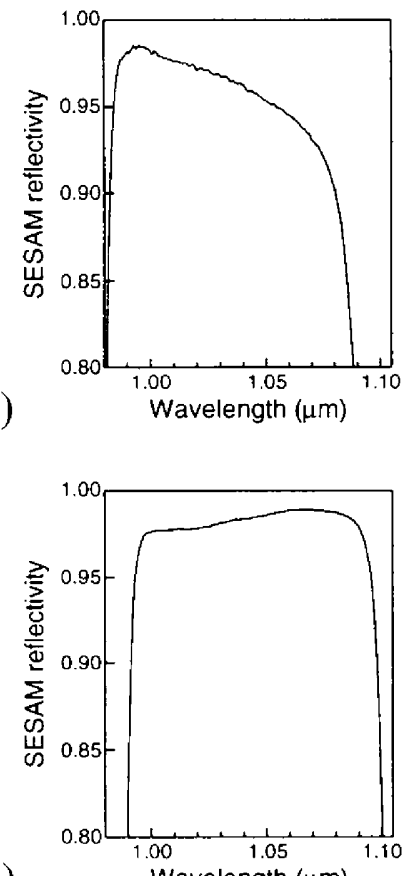

b)

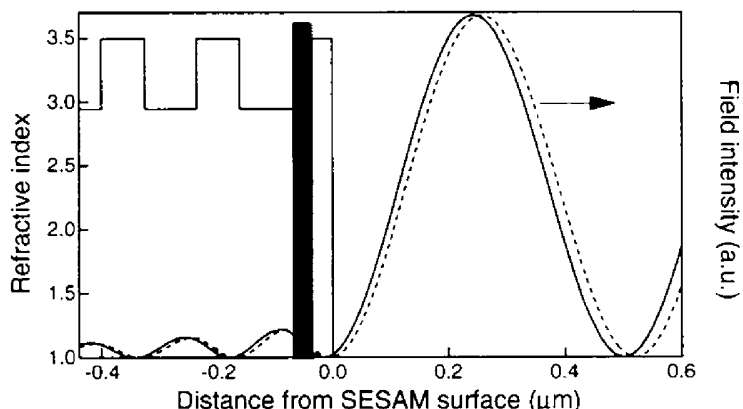

Fig. 4a,b. Reflectivity curves (left side), refractive index profile, and field intensity distribution for 1.03 (solid) and $1.05 \mu \mathrm{m}$ (dashed) wavelength (right side) for two low-finesse SESAMs. The black bars represent the absorber layers. a This SESAM (\#4) was designed to introduce higher loss at $1.05 \mu \mathrm{m}$, which was achieved by a thinner Fabry-Pérot structure, corresponding to a shorter antiresonance wavelength. The field intensity distribution does not vanish at the SESAM surface. The antiresonance thickness for 1.03 and $1.05 \mu \mathrm{m}$ wavelength is indicated (dashed line). b This SESAM (\#2 of Table 2) is nearly antiresonant for both wavelengths (node of the field intensity distribution on the surface) 
near the edge of a Bragg mirror introduces higher order dispersion, which ultimately limits the shortest obtainable pulse duration [49-51].

A second way to select the shorter wavelength is to design the Fabry-Pérot structure of the SESAM in such a way that antiresonance is obtained at $1.03 \mu \mathrm{m}$, while the $1.05 \mu \mathrm{m}$ line is closer to a resonance, where increased internal intensity leads to higher loss. Figure 4a shows this special SESAM design (\#4 in Table 3). For comparison, a classical SESAM design for antiresonance at $1.05 \mu \mathrm{m}$ is shown in Fig. 4b (for example SESAMs \#2 and \#5 to 7). We have indicated the reflectivity curves (left) as well as the refractive index profile and the field intensity distribution outside and inside the FabryPérot structures (right). Occasionally, we observed damage at the specially designed SESAM, whereas no damage occurred for the antiresonant SESAM. The suspected reason for the damage is the non-vanishing field intensity at the surface of this SESAM, as can be seen from Fig. 4a. Surfaces in general have lower damage thresholds than bulk materials [52]. This is a serious disadvantage of SESAM designs that exhibit non-vanishing field intensity at the surface.

\section{Experiments with ultrafast Yb-doped lasers}

In this section we give an overview on some previously published experimental results and also present new results on a passively modelocked high-power Yb:YAG laser. In Sect. 2.1 we discuss low-power sub-ps Yb:YAG oscillators, pumped with a Ti:sapphire laser (which could be replaced by high-brightness laser diodes). Similar oscillators based on Yb:glass, pumped with high-brightness laser diodes, are discussed in Sect. 2.2. The shortest pulses from these lasers had a duration around $60 \mathrm{fs}$, which is comparable to results achieved with Nd:glass [53]. However, Yb:glass exhibits a much broader tuning range. New results on a high-power $\mathrm{Yb}: Y A G$ laser with around 1 ps pulse duration are presented in Sect. 2.3.

\subsection{Low-power Yb:YAG oscillators}

Laser diodes with high brightness are currently available with up to $2 \mathrm{~W}$ output power at $0.94 \mu \mathrm{m}$ or $0.97 \mu \mathrm{m}$. We have used such diodes to construct Yb-doped glass lasers with low pump threshold and output powers of 50 to $500 \mathrm{~mW}$ (Sect. 2.2). The small pump and laser mode sizes in the gain medium, which can be realized with high-brightness pump sources, are favorable to suppress QML [26, 27]. As no laser diodes at $0.94 \mu \mathrm{m}$ were available at the time of the $\mathrm{Yb}: \mathrm{YAG}$

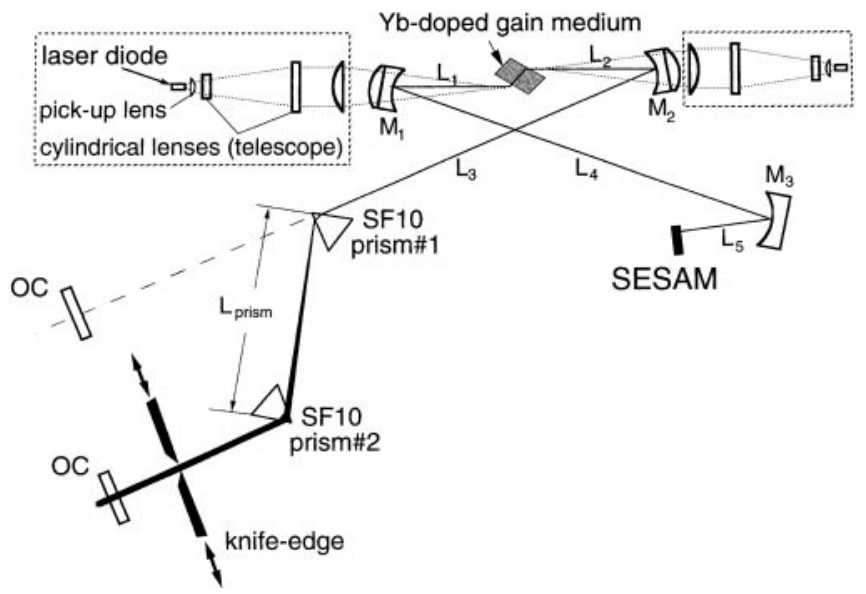

Fig. 5. Low-power cavity setup. $M_{1}=M_{2}=10 \mathrm{~cm}$ radius of curvature (ROC), $L_{1}=L_{2}=5 \mathrm{~cm}, L_{3}=40(60) \mathrm{cm}, L_{4}=80(50) \mathrm{cm}, M_{3}=7.5 \mathrm{~cm}$ ROC with the high-finesse SESAM and 20 to $40 \mathrm{~cm}$ ROC with the lowfinesse SESAM. The prism separation was $75(39) \mathrm{cm}$. The parenthetical numbers represent the values for the low-power $\mathrm{Yb}$ :glass experiments. OCs, output couplers

laser experiments [20], we used a Ti:sapphire pump laser. However, newer results on diode-pumped Yb:glass lasers demonstrate that similar performance can be obtained by pumping with high-brightness diodes despite their somewhat inferior beam quality (see also Sect. 2.2).

The typical laser setup used for the low-power experiments is shown in Fig. 5. We can operate the laser with or without dispersion compensation by inserting or removing the SF 10 prism pair [54]. All low-power Yb:YAG (and Yb:glass) lasers were operated at room temperature without actively cooling the gain medium.

We used a 3.5-mm-long, 5\%-doped Yb:YAG crystal, which was approximately twice the absorption length at our pump wavelength of $0.94 \mu \mathrm{m}$ and resulted in $\approx 85 \%$ pump absorption. The pump beam diameter (full width at $1 / \mathrm{e}^{2}$ intensity) in the Yb:YAG crystal was $\approx 100 \times 54 \mu \mathrm{m}$ in the horizontal and vertical direction, respectively. The confocal parameter of the pump beam approximately corresponded to the length of the gain medium. We designed the laser cavity in such a way that the laser mode in the gain medium matched the pump mode. Because of the proximity of pump and laser wavelength in Yb-doped materials, it is difficult to obtain good pump transmission and high reflectivity at the laser wavelength of the pump mirrors $\mathrm{M}_{1}$ and $\mathrm{M}_{2}$ at the same time. Our available mirrors had $\approx 35 \%$ reflection at the pump wavelength. Taking into account this loss, the pump power incident on the crystal was limited to $1.5 \mathrm{~W}$. The maximum pump intensity at the laser crystal was $\approx 36 \mathrm{~kW} / \mathrm{cm}^{2}$,
Table 4. Modelocked Yb:YAG lasers results. L-p, low-power experiments (see Fig. 5) H-p, high-power experiments (see Fig. 9)

\begin{tabular}{lcccccccc}
\hline Setup & $\begin{array}{c}\text { SESAM } \\
\#\end{array}$ & $\begin{array}{c}\Delta R / \\
\%\end{array}$ & Prisms & $\begin{array}{c}T_{\text {out }} / \\
\%\end{array}$ & $\begin{array}{c}P_{\text {pump }} / \\
\text { W }\end{array}$ & $\begin{array}{c}P_{\text {out }} / \\
\text { W }\end{array}$ & $\begin{array}{c}\text { Pulse width } / \\
\text { fs }\end{array}$ & $\begin{array}{c}\text { Wavelength } / \\
\mu \mathrm{m}\end{array}$ \\
\hline L-p & 1 & 0.25 & No & 2 & 1.5 & 0.16 & 1700 & 1.03 \\
L-p & 1 & 0.25 & Yes & 2 & 1.3 & 0.15 & 900 & 1.05 \\
L-p & 2 & 0.5 & Yes & 2 & 1.2 & 0.17 & 570 & 1.05 \\
L-p & 3 & 0.7 & Yes & 2 & 0.8 & 0.15 & 460 & 1.03 \\
L-p & 3 & 0.7 & Yes & 5 & 1.4 & 0.51 & 890 & 1.03 \\
L-p & 4 & 0.5 & Yes & 2 & 0.9 & 0.10 & 540 & 1.03 \\
L-p & 5 & 1.3 & Yes & 2 & 0.7 & 0.11 & 340 & 1.03 \\
H-p & 6 & 0.9 & Yes & 2 & 33 & 1.5 & 1000 & 1.03 \\
\hline
\end{tabular}


which is much higher than the pump intensity for transparency at $1.03 \mu \mathrm{m}$, which we calculated to be $\approx 1.4 \mathrm{~kW} / \mathrm{cm}^{2}$ by using (2).

The spot size on the SESAM was chosen in such a way that the intracavity pulse energy at full pump power was about 5 times the saturation energy of the SESAM.

The results obtained with the modelocked $\mathrm{Yb}$ :YAG lasers are listed in Table 4. Without dispersion compensation we achieved pulses as short as $1.7 \mathrm{ps}$ which is close to the absorber recovery time of the SESAM and can be explained with the fast saturable absorber model of Haus [40, 46]. The results with dispersion compensation can be explained with soliton modelocking $[47,48]$, which led to shorter pulses in the sub-ps regime. The time-bandwidth products of $\approx 0.33$ are close to the value of 0.315 for an ideal soliton pulse. Additionally, soliton modelocking significantly reduced the tendency towards Q-switching instabilities [27]. We believe that Kerr lens modelocking [55-58] did not contribute in our experiments because modelocking was stable and self-starting over a wide range of the resonator stability regime.

As expected from modelocking theory $[39,46]$, we achieved the shortest pulses of 340 fs by using the SESAM with the largest modulation depth. The corresponding autocorrelation trace and the spectrum are shown in Fig. 6. The spectrum covered almost the full available gain bandwidth. SESAMs with smaller modulation depth result in longer pulses and tend to operate at $1.05 \mu \mathrm{m}$. The specially designed SESAMs, as described in Sect. 1.5, resulted in operation at $1.03 \mu \mathrm{m}$ even at low output coupler transmission.

The output powers of the low-power Yb:YAG lasers ranged from 110 to $170 \mathrm{~mW}$, when we used $2 \%$ output coupler transmission. With higher output coupler transmission of $5 \%$, we obtained $0.5 \mathrm{~W}$ average output power at $1.4 \mathrm{~W}$ pump power, which corresponds to an optical efficiency of $36 \%$. However, the pulses became significantly longer ( 890 fs using SESAM \#3) because of stronger gain filtering effects at the higher saturated gain level (higher cavity loss, see Sect. 1.1).

Q-switched modelocking occurred only for pump powers below a certain critical value. This value was observed to be

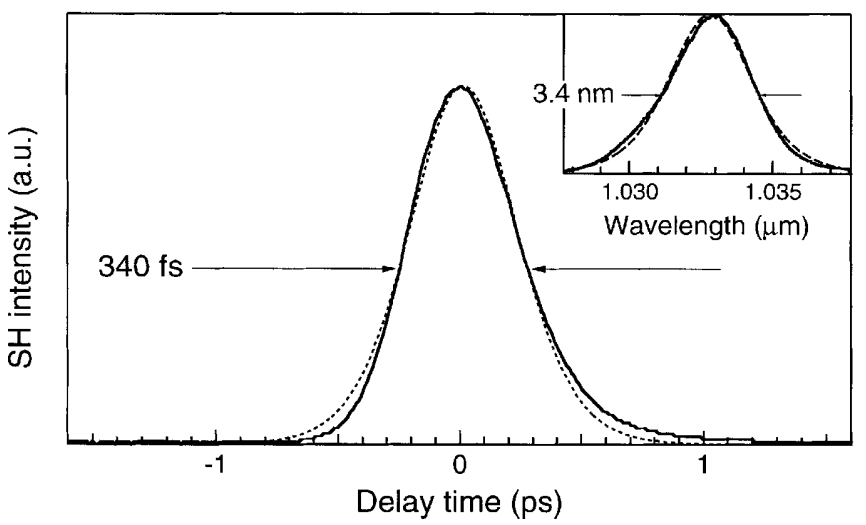

Fig. 6. Intensity autocorrelation trace and spectrum of the $340-\mathrm{fs}$ pulse obtained from a passively modelocked Yb:YAG laser. SH, second-harmonic intensity. Dotted curves: fitted soliton $\left(\mathrm{sech}^{2}\right)$ pulses

higher for large output coupler transmission and large modulation depth of the SESAM, as expected from the theory (see Sect. 1.4). However, QML could always be easily suppressed at higher pump powers.

\subsection{Low-power Yb:glass oscillators}

The basic cavity setup of our low-power Yb:glass oscillators is the same as for the low-power Yb:YAG experiments (Fig. 5). As pump sources we used two high-brightness, broad-area (30 $\mu \mathrm{m}$ ridge width) InGaAs/GaAs laser diodes (Uniphase Laser Enterprise) generating $\approx 0.9 \mathrm{~W}$ each at $0.97 \mu \mathrm{m}$. The $\mathrm{M}^{2}$ value in the slowly diverging horizontal direction was between 5 and 7, depending on the operation current of the diode. In the fast diverging vertical direction, the diode output was approximately diffraction limited. We used optimized pump mirrors $\mathrm{M}_{1}$ and $\mathrm{M}_{2}$ (Laseroptik $\mathrm{GmbH}$, Garbsen, Germany), which provided $>90 \%$ transmission at the pump wavelength of $0.97 \mu \mathrm{m}$ and $>99.8 \%$ reflectivity for wavelengths longer than $1.03 \mu \mathrm{m}$. The maximum pump power incident on the glass was $1.6 \mathrm{~W}$.

\begin{tabular}{|c|c|c|c|}
\hline Laser glass & $\begin{array}{l}\text { Phosphate } \\
\text { QX/Yb }\end{array}$ & $\begin{array}{l}\text { Silicate } \\
\text { Q-246/Yb }\end{array}$ & $\begin{array}{l}\text { Fluoride } \\
\text { phosphate }\end{array}$ \\
\hline Emission bandwidth $\Delta \lambda / \mathrm{nm}$ & 62 & 77 & 81 \\
\hline Emission cross section @ $0.975 \mu \mathrm{m} / \times 10^{-20} \mathrm{~cm}^{2}$ & 0.67 & 0.71 & 1.2 \\
\hline Emission cross section $\sigma_{\mathrm{em}}^{(L)} / \times 10^{-20} \mathrm{~cm}^{2}$ a) & 0.05 & 0.095 & 0.16 \\
\hline Absorption cross section $\sigma_{\mathrm{abs}}^{(L)} / \times 10^{-20} \mathrm{~cm}^{2}$ a) & 0.001 & 0.002 & 0.002 \\
\hline Gain saturation fluence $F_{\text {sat }, \mathrm{L}} / \mathrm{J} / \mathrm{cm}^{2}$ a) & 370 & 190 & 120 \\
\hline Fluorescence lifetime $\tau / \mathrm{ms}$ & 1.3 & 1.1 & 1.3 \\
\hline Absorption cross section $\sigma_{\mathrm{abs}}^{(P)} @ 0.97 \mu \mathrm{m} / \times 10^{-20} \mathrm{~cm}^{2}$ & 0.25 & 0.19 & 0.4 \\
\hline Emission cross section $\sigma_{\mathrm{em}}^{(P)} @ 0.97 \mu \mathrm{m} / \times 10^{-20} \mathrm{~cm}^{2}$ & 0.07 & 0.11 & 0.2 \\
\hline Pump saturation intensity $I_{\mathrm{sat}, \mathrm{P}} @ 0.97 \mu \mathrm{m} / \mathrm{kW} / \mathrm{cm}^{2}$ & 38 & 62 & 26 \\
\hline Transparency intensity $I_{\mathrm{P}, \text { trans }}^{(L)} / \mathrm{kW} / \mathrm{cm}^{2}$ & 1.3 & 2.1 & 0.5 \\
\hline $\mathrm{Yb}^{3+}$ doping density $N_{\mathrm{tot}} / \times 10^{21} \mathrm{~cm}^{-3}$ & $\approx 2$ & $\approx 1.7$ & $\approx 1.7$ \\
\hline Absorption length@0.97 $\mu \mathrm{m} / \mathrm{mm}$ & 2.0 & 3.1 & 1.5 \\
\hline Thermal conductivity $/ \mathrm{W} \mathrm{cm}^{-1} \mathrm{~K}^{-1}$ & 0.0085 & 0.013 & - \\
\hline Refractive index & 1.535 & 1.56 & $\approx 1.5$ \\
\hline Nonlinear refractive index $/ \times 10^{-16} \mathrm{~cm}^{2} / \mathrm{W}$ & 3.2 & 3.8 & - \\
\hline
\end{tabular}

a) at $1.06 \mu \mathrm{m}$ wavelength 
We focused the pump beam to a spot diameter of $\approx 90 \mu \mathrm{m} \times 50 \mu \mathrm{m}$ in the glass in the horizontal and vertical direction, respectively. The confocal length inside the glass was $12 \mathrm{~mm}$ in the vertical and $3 \mathrm{~mm}$ in the horizontal direction. The maximum pump intensity was $\approx 44 \mathrm{~kW} / \mathrm{cm}^{2}$, which was much higher than the transparency intensity of the Yb-doped glasses. The transparency intensity as well as many other important parameters of the different $\mathrm{Yb}$-doped glasses is listed in Table 5. All glasses were highly doped $(\approx 15 \mathrm{wt} . \%$ $\mathrm{Yb}_{2} \mathrm{O}_{3}$ ) in order to provide short absorption lengths.

Because of the low emission cross sections, we could not avoid Q-switched modelocking when we operated the lasers without dispersion compensation. However, when we inserted a prism pair for dispersion compensation, QML occurred only for lower pump powers. This can be explained with soliton effects [27].

Stable self-starting soliton modelocking was obtained in all cases. KLM was not expected to occur, and the characteristic sensitivity to variations of cavity parameters was not observed. The results of our low-power Yb:glass experiments are listed in Table 6. The shortest pulse duration was $\approx 60$ fs for all glasses (Fig. 7) and was achieved at low output coupler transmissions of 1 to $2 \%$. The output power in these experiments was between 50 and $65 \mathrm{~mW}$. Higher output power and laser efficiency was achieved with increased output coupler transmission, accepting somewhat longer pulse duration because of the stronger effect of the gain filter (see Sect. 1.1). The highest modelocked output power of $0.38 \mathrm{~W}$ was achieved with the phosphate glass. The FWHM pulse duration was $120 \mathrm{fs}$.

Both of the other glasses showed lower efficiency than the phosphate glass. In the case of the silicate glass, one reason was that our pump and laser setup was optimized for glass lengths of about $3 \mathrm{~mm}$ (confocal length of the pump beam). This was optimal for the phosphate glass with its absorption length of $\approx 2 \mathrm{~mm}$. However, the silicate glass had a slightly longer absorption length of $3.2 \mathrm{~mm}$ which resulted in lower pump absorption. Another reason might be energy transfer to thulium impurities, which was indicated by some blue fluorescence.

As the only Yb-doped glass that we investigated, the fluoride phosphate exhibited thermal problems. This led to ther-

Table 6. Modelocked low-power Yb:glass laser results

\begin{tabular}{lccc}
\hline Glass & $\begin{array}{c}\text { Phosphate } \\
\text { QX/Yb }\end{array}$ & $\begin{array}{c}\text { Silicate } \\
\text { Q-246/Yb }\end{array}$ & $\begin{array}{c}\text { Fluoride } \\
\text { phosphate }\end{array}$ \\
\hline SESAM \# & 6 & 7 & 6 \\
Shortest pulse width /fs & 60 & 60 & 60 \\
Center wavelength / $\mu \mathrm{m}$ & 1.058 & 1.08 & 1.057 \\
Output power /mW & 65 & 53 & 50 \\
Pump power /mW & 700 & 700 & 750 \\
Highest cw power /mW & 510 & 300 & 70 \\
Highest modelocked power $/ \mathrm{mW}$ & 380 & 200 & 50 \\
Pump power /mW & 1600 & 1600 & 750 \\
Pulse width /fs & 120 & 100 & 60 \\
Center wavelength / $\mu \mathrm{m}$ & 1.05 & 1.07 & 1.057 \\
Tuning range / $\mu \mathrm{m}$ & $1.025-1.065$ & $1.03-1.082$ & $1.055-1.069$ \\
Pulse widths $/ \mathrm{fs}$ & $115-350$ & $110-450$ & $60-160$ \\
Output powers $/ \mathrm{mW}$ & $90-240$ & $60-150$ & $20-50$ \\
Pump power $/ \mathrm{mW}$ & 1200 & 1200 & 750 \\
\hline
\end{tabular}
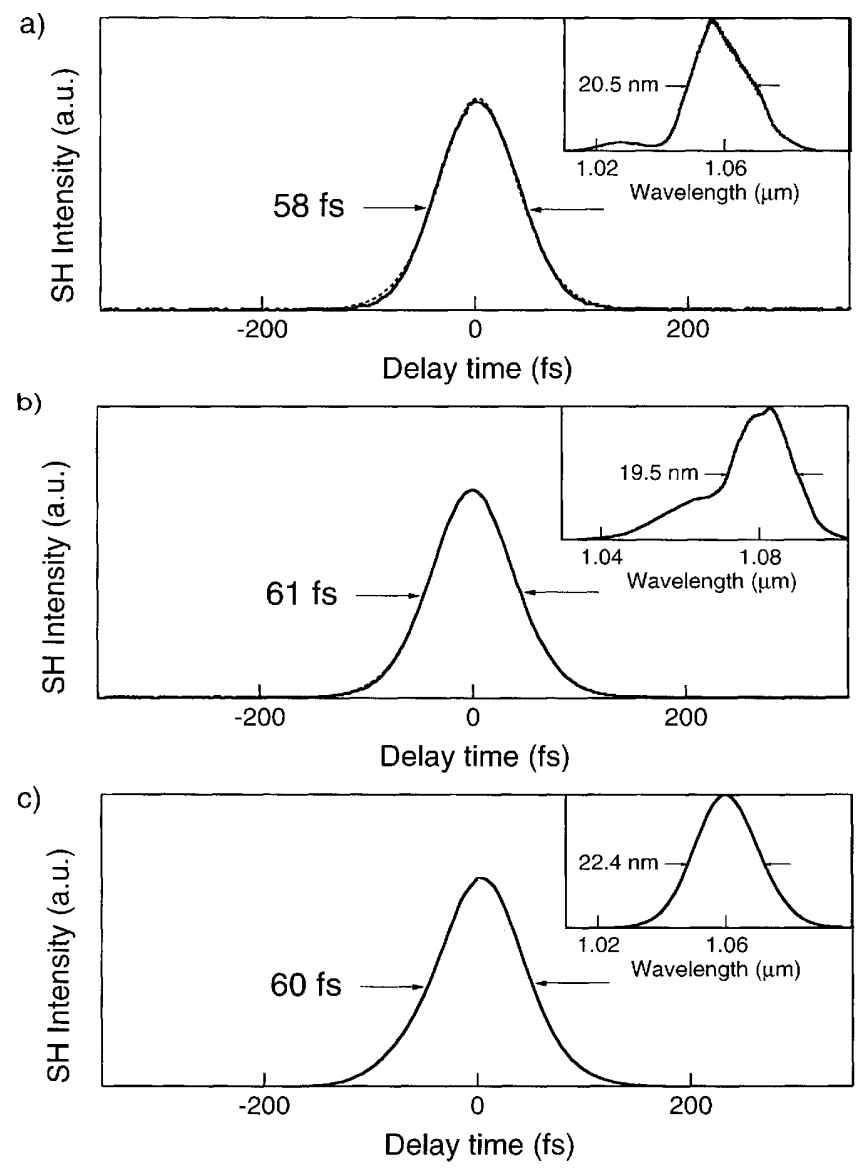

Fig. 7a-c. Shortest pulse intensity autocorrelation traces (SH: second harmonic) and spectra (insets) obtained from a the $\mathrm{QX} / \mathrm{Yb}$ phosphate (SESAM \#6), b the Q-246/Yb silicate (SESAM \#7), and c the fluoride phosphate (SESAM \#6) glass laser

mal fracture at pump powers higher than $0.8 \mathrm{~W}$. Poor optical quality of the glass was also visible.

We achieved tuning of the modelocked pulses by inserting a knife-edge in the spatially dispersed beam near the output coupler. The tuning ranges are listed in Table 6 and indicated in Fig. 8 for each glass together with the measured emission and absorption cross sections. The tuning ranges are significantly larger than the maximum modelocked bandwidth. The reason for this is that the amplification bandwidth for any given inversion level is limited (see Fig. 2), whereas tuning with the knife-edge modifies the inversion level and thus shifts the wavelength of maximum gain.

\subsection{High-power diode-pumped ultrafast $\mathrm{Yb}: \mathrm{YAG}$ laser}

While high-brightness laser diodes are currently limited to about $2 \mathrm{~W}$ pump power, up to $40 \mathrm{~W}$ is available from highpower diode bars, promising multiwatt output powers from Yb-doped lasers. The main challenge, however, is to cope with the poor beam quality from such pump sources. This leads to lower pump intensities and, thus, lower efficiency of quasi-three-level lasers. The output of our 40-W diode bar (DILAS GmbH, Germany) is a highly elliptical beam with $M_{\text {slow }}^{2}=3000$ and $M_{\text {fast }}^{2}=10$ in the horizontal and the vertical direction, respectively. The strongly different beam quality in 

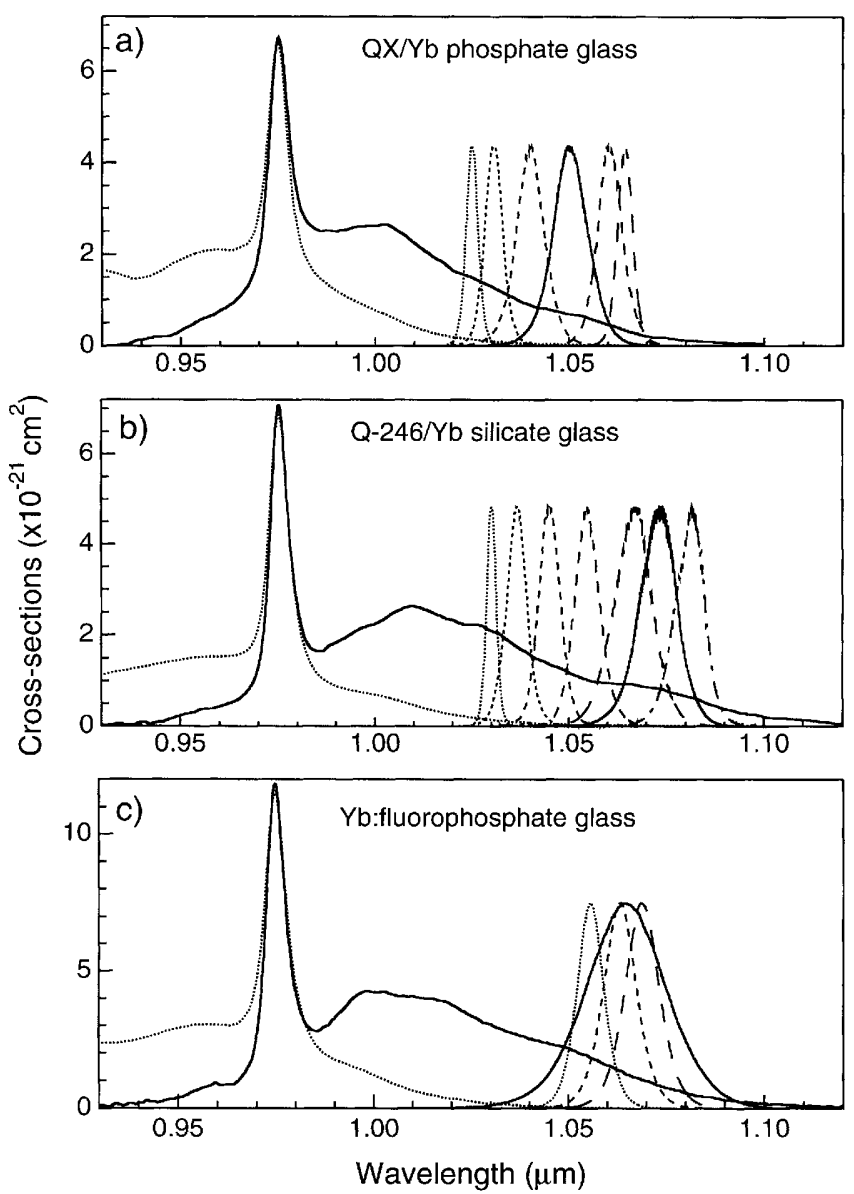

Fig. 8a-c. Measured emission and absorption spectra of the different $\mathrm{Yb}$ glass materials (see Table 5). The tuning range is visualized by showing some measured pulse spectra. a QX/Yb phosphate glass, b Q-246/Yb silicate glass, $\mathbf{c}$ Yb-doped fluoride phosphate glass

both directions constitutes a second challenge, as the pump beam must be matched to the laser mode in the resonator. Simply focusing the pump beam to a circular spot is not favorable because the minimum spot size would be determined by the poor beam quality in the horizontal direction while the much better beam quality in the vertical direction would not be fully exploited. The resulting pump intensity would be too low. There are two ways to solve this problem. The first is to equalize the beam qualities in both directions with a special beam shaper $[59,60]$, which allows for the generation of a circular pump beam. The second [61-63] is to apply optimum focusing in both directions, i.e. generate a beam with the minimum acceptable confocal parameter in both directions. The cavity mode must then be matched to the resulting highly elliptical pump beam. We have applied the second approach because of the superior thermal properties. Efficient cooling is possible by the use of a rather thin laser crystal, reducing the maximum temperature excursion in the gain medium. This is beneficial for the efficiency of an Yb:YAG laser. Moreover, the nearly one-dimensional heat flow reduces the effects of thermal lensing and stress-induced birefringence.

Our 0.94- $\mu \mathrm{m}, 40-\mathrm{W}$ diode bar was delivered with a cylindrical micro-lens close to the output face of the diode, which resulted in a nearly collimated beam in the vertical direction. We used two more cylindrical lenses for further collimating the vertical direction as well as for collimating the horizontal direction. Because of the large extension of the pump beam in the horizontal direction, we used optics with large apertures of 2 inches, still causing $\approx 18 \%$ loss of pump power due to clipping. To focus the asymmetric pump beam into the gain medium, we used a pair of two closely located $80-\mathrm{mm}$ lenses to achieve a focal length of $\approx 40 \mathrm{~mm}$. We measured the pump spot diameter (full width at $1 / \mathrm{e}^{2}$ intensity) to be $2.8 \mathrm{~mm} \times 0.18 \mathrm{~mm}$ in the horizontal and vertical directions, respectively. The confocal parameter in the horizontal (vertical) direction was measured to be $4 \mathrm{~mm}(5.5 \mathrm{~mm})$ in air, corresponding to $\approx 7 \mathrm{~mm}(\approx 10 \mathrm{~mm})$ in the crystal. All lenses of the pump optics were AR-coated at $0.94 \mu \mathrm{m}$. We obtained a maximum power of $33 \mathrm{~W}$ at the location of the laser crystal and a pump intensity of $\approx 6.5 \mathrm{~kW} / \mathrm{cm}^{2}$, to be compared with the intensity of $1.4 \mathrm{~kW} / \mathrm{cm}^{2}$ for transparency at $1.03 \mu \mathrm{m}$.

The cavity setup of our high-power Yb:YAG laser is shown in Fig. 9. We achieved mode matching between the laser mode and the elliptical pump beam by using a cylindrical curved cavity mirror next to the crystal. The folded resonator mode inside the crystal results in a doubled gain per round trip compared to a standard end-pumped cavity setup. The cavity length of $3.3 \mathrm{~m}$ results in a pulse repetition rate of $45 \mathrm{MHz}$.

We used a flat/Brewster cut Yb:YAG crystal with 3 at. \% Yb-doping. The absorption length was $\approx 3 \mathrm{~mm}$ at our pump wavelength of $0.94 \mu \mathrm{m}$. The end face of the crystal was coated for $\approx 90 \%$ transmission at the pump wavelength and $\approx 99.8 \%$ reflectivity at the laser wavelength of $1.03 \mu \mathrm{m}$. The optimum path length in the gain medium, adjustable by lateral translation of the flat/Brewster cut Yb:YAG crystal, was found to be $\approx 3 \mathrm{~mm}$. The optimum path length (as well as the pump absorption efficiency of about 63\%) was only weakly dependent on the output coupler transmission because the ground state depletion was never higher than $7 \%$.

For efficient heat removal, the Yb:YAG crystal was chosen to be only $0.8 \mathrm{~mm}$ thick. It was mounted between two

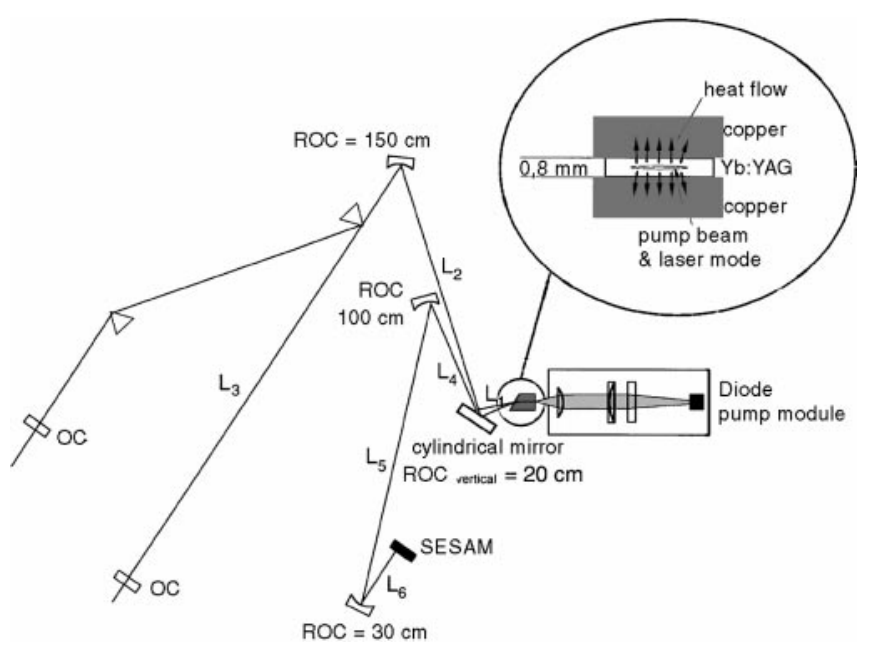

Fig. 9. High-power cavity setup. $L_{1}=12.3 \mathrm{~cm}, L_{2}=98 \mathrm{~cm}, L_{3}=97 \mathrm{~cm}$, $L_{4}=47 \mathrm{~cm}, L_{5}=60 \mathrm{~cm}$, and $L_{6}=13 \mathrm{~cm}$ with the $30-\mathrm{cm}$ ROC mirror. The prism separation was $53.5 \mathrm{~cm}$. OC, output coupler. Inset: schematic crosssectional view of the laser crystal pumped by the strongly asymmetrical diode beam. The crystal geometry causes a mainly one-dimensional heat flow to the copper heat sinks 
thermo-electric-cooled copper heat sinks whose minimum temperature was limited to $\approx 5^{\circ} \mathrm{C}$ by the onset of water condensation. Assuming a purely one-dimensional heat flow and perfect heat flow across the interface between crystal and heat sink, we estimate the temperature in the center of the crystal to rise by only about $2 \mathrm{~K}$ above the heat sink temperature per watt of dissipated power. The importance of keeping the crystal temperature low is confirmed by the observation that the threshold power increases by about $3 \mathrm{~W}$ when the heat sink temperature is increased by $25 \mathrm{~K}$.

In a cw configuration, i.e., without prisms in the cavity and the SESAM replaced by a high reflecting mirror, we obtained the maximum output power of $4.3 \mathrm{~W}$ from an incident power of $33 \mathrm{~W}$ using an output coupler with $15 \%$ transmission. The threshold power and slope efficiency with respect to incident power on the crystal were $15 \mathrm{~W}$ and $24 \%$, respectively.

To obtain modelocked operation, we inserted a SESAM into the cavity (Fig. 9). Various measures had to be taken to suppress the tendency for Q-switched modelocking. This tendency is strong due to the large laser mode area (enforced by the poor pump beam quality) and the low cross sections of Yb:YAG. Both the multiple paths through the gain medium and the relatively low repetition rate were favorable for the suppression of QML. Furthermore, the mode area on the absorber was kept rather small $\left(A_{\text {eff,A }}=7 \times 10^{-4} \mathrm{~cm}^{2}\right)$, limited by the onset of multiple pulsing [40]. The intracavity pulse energy was increased by using an output coupler with only $3 \%$ transmission, although this compromised the power efficiency. Despite all these measures, QML could only be suppressed in soliton modelocked operation, using a pair of SF10 prisms for dispersion compensation. In this way, we obtained cw modelocking with soliton-like 1-ps pulses (Fig. 10) and $1.5 \mathrm{~W}$ average output power. This power is just the same as that recently achieved with Nd:glass in a similar configuration [64].

We believe that the low optical efficiency of only $5 \%$ in modelocked operation can be significantly improved in the future. We plan to double the pump power and pump intensity by using two polarization-coupled 40-W diode bars. Thermal problems are not expected due to the efficient cooling arrangement. The higher pump intensity should increase the efficiency by operating further above threshold as well as by using a higher output coupler transmission and a longer path length in the gain medium. Nitrogen purging of the crystal mount should allow for better cooling and, thus, reduce the threshold power.

Ongoing research in the field of high-power, highbrightness laser diodes will probably soon provide very efficient laser diodes, which are ideally suited to pump Yb-doped solid-state lasers. Recently, $10 \mathrm{~W} \mathrm{cw}$ output power from a $100-\mu \mathrm{m}$-aperture Al-free laser diode at a wavelength of $0.97 \mu \mathrm{m}$ was demonstrated $[65,66]$. With these high-power, high-brightness laser diodes it becomes easier to achieve efficient and stable passively modelocked operation with multiwatt output powers, possibly even with Yb:glass despite the lower cross sections. This is not possible with the currently available high-power low-brightness diode bars, for which it is already a challenge to suppress QML in a Yb:YAG laser.



Fig. 10. Intensity autocorrelation trace and spectrum of the 1-ps pulse obtained from the high-power soliton modelocked Yb:YAG laser. The average output power was $1.5 \mathrm{~W}$. SH, second-harmonic intensity

\section{Ultrafast Yb-doped laser amplifiers}

\subsection{Short-pulse amplification in Yb-doped and $\mathrm{Nd}$-doped amplifiers}

The pulse energy available from modelocked oscillators is limited to moderate values by the limited pump power and the high repetition rate of typically around $100 \mathrm{MHz}$. Much higher pulse energies can be obtained with regenerative amplifiers. Here a single seed pulse of moderate energy, typically extracted from a modelocked oscillator, is injected into the amplifier cavity with a Pockels cell, circulated there until the gain is saturated, and finally extracted again with the Pockels cell. The extractable energy can be a significant fraction of the total energy initially stored in the amplifier gain medium. The most important parameters for a regenerative laser amplifier are the round-trip small-signal gain coefficient $g_{0}$, the gain saturation energy $E_{\mathrm{sat}, \mathrm{L}}=F_{\mathrm{sat}, \mathrm{L}} A_{\mathrm{eff}, \mathrm{L}}$, and the round-trip loss coefficient $l$.

The stored energy in the amplifier medium is related to the small-signal gain and to the gain saturation energy. For the stored energy in a quasi-three-level amplifier material, we can derive the expression

$E_{\text {stored }}=L_{\mathrm{g}} A_{\text {eff, } \mathrm{L}} N_{2} h v_{L}=E_{\text {sat, } \mathrm{L}}\left(g_{0}+2^{*} L_{\mathrm{g}} N_{\text {tot }} \sigma_{\text {abs }}^{(L)}\right)$.

For a cw-pumped gain medium, the stored energy is limited by the pump power. In steady state, the excitation rate equals the spontaneous emission, and we have

$E_{\text {stored }}=\eta_{\text {parasitic }} \eta_{\mathrm{Q}} \eta_{\mathrm{P}} P_{\text {pump }} \tau$.

Here, $P_{\text {pump }}$ denotes the incident pump power. The absorbed fraction of the pump power is represented by $\eta_{\mathrm{P}}$, which additionally accounts for geometrical effects, as for example, imperfect overlap of the pumped region with the amplifier mode. $\eta_{\mathrm{Q}}=\lambda_{\mathrm{P}} / \lambda_{\mathrm{L}}$ denotes the quantum defect of the used amplifier material, and $\eta_{\text {parasitic }} \leq 1$ accounts for loss processes such as energy transfer to impurities in the amplifier crystal, upconversion, or excited-state absorption.

Comparing $\mathrm{Yb}$ and $\mathrm{Nd}$ as dopants, we find that $\mathrm{Yb}$-doped gain media can store significantly more energy because of the longer fluorescence lifetime and higher pump efficiency, provided that the pump absorption efficiency is high. In a quasithree-level medium, the latter is limited by the available pump 
intensity because the pump intensity at the end of the medium should reach the intensity needed for transparency. Therefore the better energy storage capability of Yb-doped media can only be exploited with a pump source of sufficiently high brightness. Considering Yb:YAG for pumping at $0.94 \mu \mathrm{m}$ and operation at $1.03 \mu \mathrm{m}$, we find that this condition is well fulfilled with high-brightness pump diodes. With high-power low-brightness diode bars, the advantage of $\mathrm{Yb}$ over $\mathrm{Nd}$ is smaller. However, the better efficiency of $\mathrm{Yb}$ (due to the low quantum defect and the absence of quenching processes) is favorable because of the reduced tendency for thermal lensing and fracture.

The extractable pulse energy is given by the product of stored energy and the extraction efficiency. The latter is limited by cavity losses, particularly if the gain is low, because this increases the required number of passes through the amplifier. In addition, even a lossless quasi-three-level medium does not allow for total energy extraction because gain is available only as long as the stored energy exceeds the level necessary for transparency. Here again the brightness of the pump source is a limiting factor. With sufficient brightness, a good extraction efficiency is achieved because the stored energy can be far above the energy for transparency, and the high gain reduces the required number of passes. An extensive theoretical and numerical treatment of multi-pass amplification in an amplifier with round-trip loss coefficient $l$ can be found in [67]. For small values of the cavity-loss coefficient and the small-signal gain coefficient, a rate-equation analysis yields the following result for the maximum extractable pulse energy:

$$
\hat{E}_{\mathrm{P}, \text { extr }}=E_{\text {sat,L }}\left\{g_{0}-l\left[1+\ln \left(g_{0} / l\right)\right]\right\}+E_{0} .
$$

Here, $E_{0}$ denotes the initial pulse energy which, in case of a regenerative amplifier, is the seed pulse energy.

In Fig. 11 we compare regenerative amplifiers based on Yb:YAG (operating at $1.03 \mu \mathrm{m}$ ) and Nd:YAG. The material parameters were taken from Table 1. We assume pumping with high-power low-brightness diode bars and set the effective laser mode area $A_{\text {eff.L }}=4 \times 10^{-3} \mathrm{~cm}^{2}$, which was approximately the mode size in our high-power Yb:YAG os-

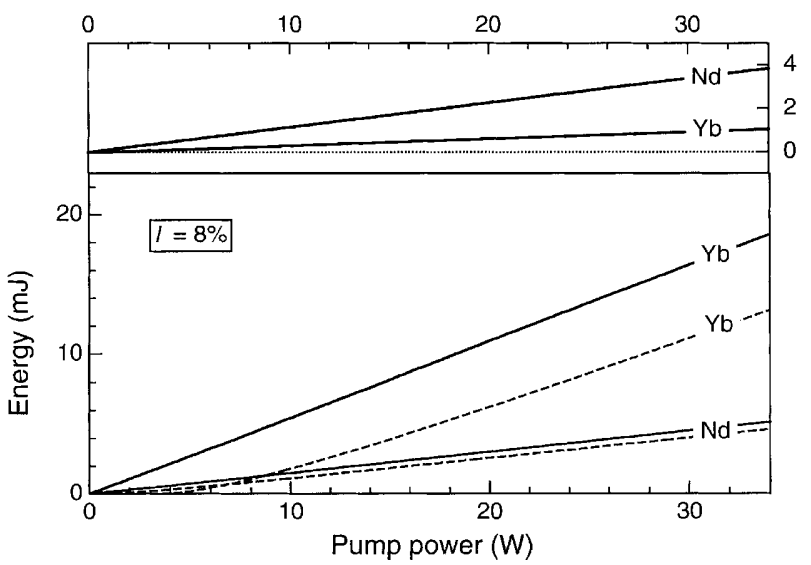

Fig. 11. Pulse energy and small-signal gain coefficient versus pump power according to relations (6) to (8) for regenerative amplifiers based on $\mathrm{Yb}$ :YAG and Nd:YAG $\left(A_{\mathrm{eff}, \mathrm{L}}=4 \times 10^{-3} \mathrm{~cm}^{2}\right)$. The stored pulse energy is represented by the solid lines, while the extracted energy is visualized by the dashed curves (assumed round-trip loss: 8\%) cillator. To account for the transparency condition at the laser wavelength in quasi-three-level lasers (see Sect. 1.2), we assumed only $63 \%$ pump absorption (one absorption length) for Yb:YAG, while we set the pump absorption for the Nd:YAG crystal to $86 \%$. Parasitic effects such as excited-state absorption are neglected in both cases, although these can be significant in Nd:YAG, particularly for high inversion levels. In the upper part of the figure, the small-signal gain coefficient is plotted as a function of pump power by combining relations (5) and (6). The solid curves in the bottom part show the stored energy versus pump power, while the dashed curves represent the maximum extractable energy according to relation (7) for an assumed loss coefficient of $8 \%$. Here we have neglected the initial pulse energy $E_{0}$, which typically is several orders of magnitude lower than the finally extracted energy in regenerative amplifiers. At low pump powers, the Nd:YAG amplifier results in higher extracted pulse energies because in this regime the Yb:YAG amplifier suffers from its quasi-three-level nature. Above $\approx 8 \mathrm{~W}$ the extractable pulse energy from the Yb:YAG amplifier exceeds that of the Nd:YAG amplifier, as expected from the significantly higher stored energy. For example, we could extract $11 \mathrm{~mJ}$ from the Yb:YAG amplifier compared to only $4 \mathrm{~mJ}$ from Nd:YAG when the pump power equals $30 \mathrm{~W}$. The advantage of $\mathrm{Yb}$ :YAG is significantly more pronounced if we use high-brightness laser diodes instead of the low-brightness ones.

It is also interesting to compare the extraction efficiencies of the two amplifiers, which are defined as

$\eta_{\text {extr }}=\frac{E_{\mathrm{P} \text {,extr }}}{E_{\text {stored }}}=\frac{g_{0}-l\left[1+\ln \left(g_{0} / l\right)\right]}{g_{0}+2 * L_{\mathrm{g}} N_{\mathrm{tot}} \sigma_{\mathrm{abs}}^{(L)}}$.

Here we have already neglected the term with the seed pulse energy (see (7)). In Fig. 12 we present the extraction efficiency versus the pump power for various values of the loss coefficient for the same Yb:YAG and Nd:YAG amplifiers as in Fig. 11. As discussed, even at zero loss the extraction efficiency of the quasi-three-level amplifier is lower than unity, whereas, in this hypothetical case, the efficiency of the ideal four-level amplifier would be 1 (upper dashed line). We can conclude that it is important to minimize the round-trip loss in the regenerative amplifier cavity in order to achieve good

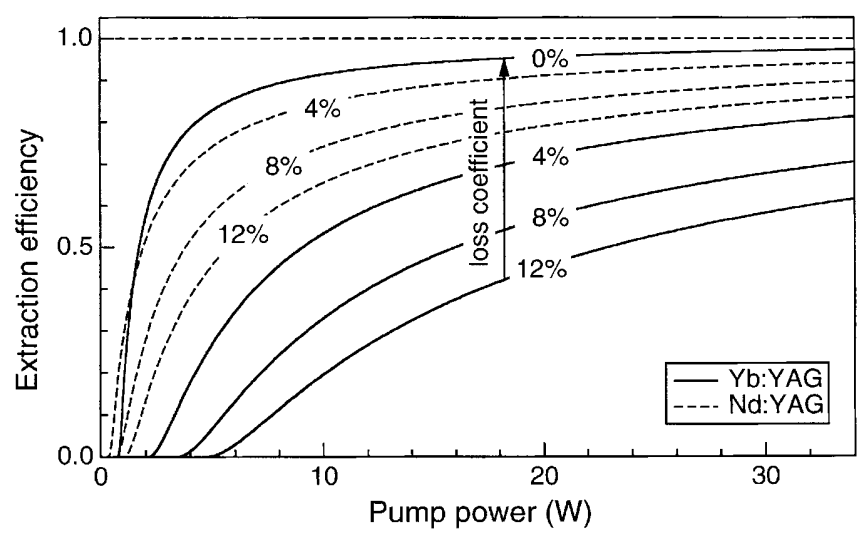

Fig. 12. Extraction efficiency for an Yb:YAG (solid) and a Nd:YAG (dashed) regenerative amplifier for various values of the round trip loss. The lowest curves correspond to $12 \%$ loss, followed by $8 \%, 4 \%$, and finally $0 \%$ 
extraction efficiency especially for Yb-doped amplifiers with their lower gain. This is because the lower gain in $\mathrm{Yb}$-doped amplifiers requires more passes through the amplifier to saturate the gain and extract the stored energy. For pumping with high-brightness diodes the extraction efficiency for $\mathrm{Yb}: \mathrm{YAG}$ amplifiers is much more comparable to Nd:YAG amplifiers.

Another important issue in regenerative amplifiers is the repetition frequency of the amplification process, which determines the average output power of the regenerative amplifier. Full recovery of the gain after extraction of a pulse takes a time on the order of the fluorescence lifetime $\tau$. Operation of a regenerative amplifier at a repetition rate higher than the inverse fluorescence lifetime results in a lower stored energy and, consequently, in a roll-off in the extracted energy. Therefore, the larger energy storage capability of $\mathrm{Yb}$-doped media is realized only at 3 to 4 times lower repetition rates compared to Nd-doped media.

The limited bandwidth of the gain medium is of particular importance in a regenerative amplifier because of the high gain seen by the pulse in many passes through the medium. This can lead to significant spectral narrowing and a corresponding increase of pulse duration $[68,69]$. For an estimate, we assume a Gaussian pulse with FWHM (full width at half maximum) duration $\tau_{\mathrm{P} \text {,in }}$, which passes $k$ times through an amplifier with single-pass gain coefficient $g_{0}$ and a parabolically approximated gain shape with FWHM bandwidth $\Delta f_{\mathrm{a}}$. The resulting pulse width is

$\tau_{\mathrm{P}, \text { out }}=\tau_{\mathrm{P}, \text { in }}\left[1+0.28 \frac{k g_{0}}{\Delta f_{\mathrm{a}}^{2} \tau_{\mathrm{P}, \mathrm{in}}^{2}}\right]$.

Comparing Yb:YAG and Nd:YAG regenerative amplifiers with $60 \mathrm{~dB}$ gain, we find that a pulse with an initial duration of $1 \mathrm{ps}$ would result in an output pulse of $2.5 \mathrm{ps}$ and $26 \mathrm{ps}$ width, respectively. Both the approximately 10 times shorter amplified pulse duration and the significantly higher achievable pulse energy illustrate the potential of Yb:YAG to generate high peak powers.

\section{$3.2 \mathrm{Yb}: Y A G$ regenerative amplifier experiment}

We have recently demonstrated the first diode-pumped Yb:YAG regenerative amplifier [31], which was seeded with $750 \mathrm{fs}, 140 \mathrm{pJ}$ pulses at $1.03 \mu \mathrm{m}$ from a passively modelocked

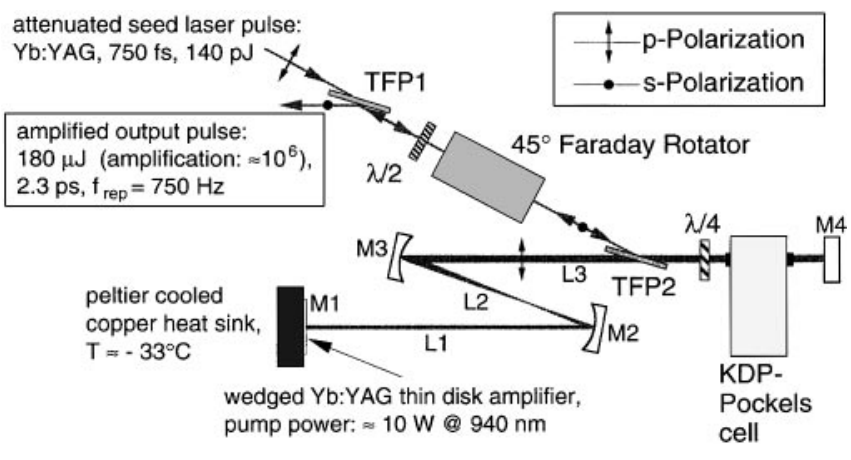

Fig. 13. Diode-pumped $\mathrm{Yb}: \mathrm{YAG}$ regenerative amplifier setup. $M_{1}$ is a Yb:YAG thin disk used as "active" mirror; $L_{1}=47 \mathrm{~cm}, L_{2}=32.7 \mathrm{~cm}$, $L_{3}=62 \mathrm{~cm}, M_{2}$ and $M_{3}$ have $20 \mathrm{~cm}$ and $40 \mathrm{~cm}$ ROC, respectively. TFPs are thin film polarizers, $\lambda / 4$ is a quarter-wave plate, $\lambda / 2$ is a half-wave plate
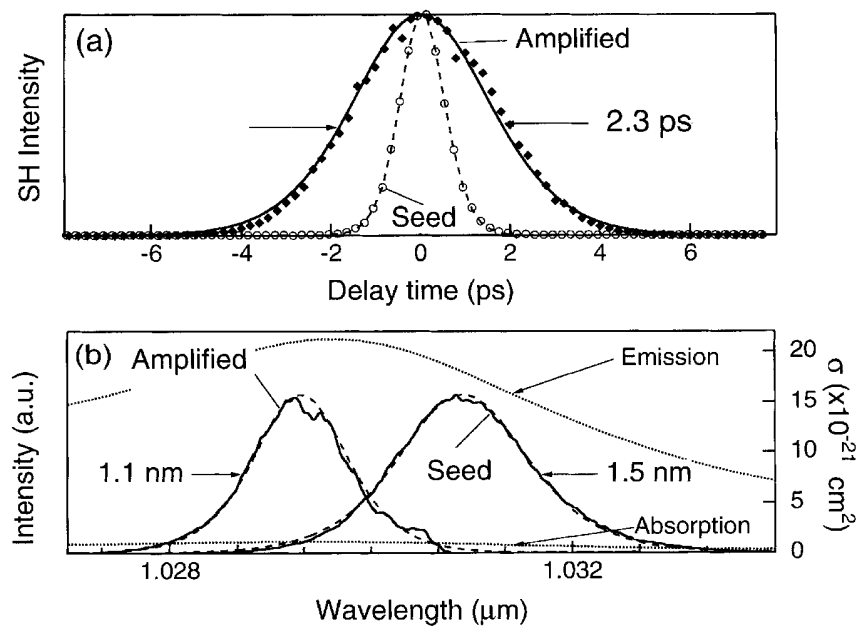

Fig. 14. a Normalized slow-scan autocorrelations and fits (assuming hyperbolic secans pulse shape) of the 750-fs seed pulses (circles) and the 2.3-ps amplified pulses (squares). b Measured (solid) and $\operatorname{sech}^{2}$-fitted (dashed) pulse spectra of seed (right) and amplified (left) pulse showing the effect of gain narrowing and the weaker re-absorption in the amplifier (blueshift). For comparison, we have indicated the emission and absorption curve of $\mathrm{Yb}: \mathrm{YAG}$

Yb:YAG oscillator similar to the one described in Sect. 2.1. The cavity setup of the regenerative amplifier is shown in Fig. 13. The amplifier head was based on the thin-disk concept [7]. The 300- $\mu \mathrm{m}$ thin gain medium was pumped by $\approx 8 \mathrm{~W}$ incident power of which about $80 \%$ were absorbed. From (6), we calculated a stored energy of $\approx 3.7 \mathrm{~mJ}$. The pump intensity of $\approx 4.4 \mathrm{~kW} / \mathrm{cm}^{2}$ resulted in a maximum excitation level of $14 \%$ and a measured small-signal gain coefficient of $\approx 0.26$. For a round-trip loss coefficient of 0.08 (cumulated loss from the polarizer and the Pockels cell), we calculated a maximum extractable pulse energy of approximately $450 \mu \mathrm{J}$ by using (7). In the experiment, we extracted a lower pulse energy of $270 \mu \mathrm{J}$ corresponding to an extraction efficiency of $7 \%$. Owing to $\approx 33 \%$ loss during extraction, we obtained a reduced pulse energy of $180 \mu \mathrm{J}$ at the amplifier output. The repetition rate was $750 \mathrm{~Hz}$. Significantly higher extraction efficiency could be achieved by reducing the round-trip loss in the amplifier cavity. For example, a reduction of the round-trip loss to $6 \%$ should already double the extraction efficiency.

Because of the $\approx 60 \mathrm{~dB}$ pulse amplification, gain narrowing broadened the pulse width to $2.3 \mathrm{ps}$, which is in good agreement with a calculation based on (9) (see Fig. 14). During amplification, the pulse spectrum narrows and shifts to shorter wavelengths (Fig. 14b). The wavelength shift is caused by the higher average inversion level (and thus shorter peak gain wavelength) of the amplifier compared to the oscillator (see Sect. 1.1).

The output pulse energy of $180 \mu \mathrm{J}$ in a 2.3 -ps short pulse resulted in a peak power as high as $\approx 80 \mathrm{MW}$. By reducing the high cavity loss or by increasing the pump power, while still maintaining a small spot area in the gain medium, it should be possible to increase the extracted energy from a Yb:YAG amplifier to the millijoule level (see Sect. 3.1). There is a significant potential to reduce the cavity loss by using polarizers and Pockels cells with higher optical quality.

Previously, $88-\mu \mathrm{J}$ pulses with 11 ps duration ( $8 \mathrm{MW}$ peak power) were reported from a regenerative amplifier based on 
$\mathrm{Nd}$ :YLF [70] and 1.3-mJ pulses with $25 \mathrm{ps}$ duration (50 MW peak power) [32] were obtained from a Nd:YAG regenerative amplifier. Higher pulse energies of $750 \mu \mathrm{J}$ [71] and $2.5 \mathrm{~mJ}$ [72] were obtained from Nd:YLF amplifiers by using high pump powers of $15 \mathrm{~W} \mathrm{cw}$ and $120 \mathrm{~W}$ quasi-cw, respectively. The output pulse widths were not measured. Another $\mathrm{Nd}$ :YAG regenerative amplifier pumped with $80-\mathrm{mJ}$ pump pulses at $50 \mathrm{~Hz}$ repetition rate produced 6-mJ pulses with approximately $1 \mathrm{~ns}$ pulse duration which resulted in a peak power of $\approx 6 \mathrm{MW}[73]$.

Our results with the Yb:YAG regenerative amplifier $(180 \mu \mathrm{J}, 2.3 \mathrm{ps}, 80 \mathrm{MW})$ show that this material is superior for high peak power generation because of the significantly shorter achievable amplified pulse widths due to reduced gain narrowing. As discussed above, millijoule pulse energies should be possible with pulse durations of a few picoseconds and peak powers on the gigawatt level, possibly even without resorting to chirped pulse amplification.

\subsection{Yb:phosphate glass chirped pulse regenerative amplifier experiments}

Yb-doped glass materials exhibit a bandwidth which is broad enough to support pulses as short as $60 \mathrm{fs}$. Due to the high peak intensities associated with ultrashort pulses in the $100 \mathrm{fs}$ regime, the technique of chirped pulse amplification (CPA) [74] must be used in order to prevent damage of the optical components inside the amplifier cavity. CPA has been demonstrated in a laser-pumped Yb-doped fiber [75] and in bulk Yb:glass [76].

We have recently demonstrated the first directly diodepumped Yb:phosphate glass (Kigre QX/Yb) chirped pulse amplifier [77]. As an amplifier cavity, we used a similar setup as for the laser shown in Fig. 5. We only substituted the curved focusing mirrors next to the gain medium by $7.5-\mathrm{cm}$ ROC mirrors, replaced the SESAM by a highly reflecting mirror, and inserted a prism polarizer and a lithium niobate Pockels cell to inject and eject the pulses. We pumped the Yb:glass amplifier medium with $1.2 \mathrm{~W}$ from two high-brightness diodes. The 200-fs seed pulses from a modelocked Ti:sapphire laser tuned to $1.04 \mu \mathrm{m}$ were stretched to 1 to $2 \mathrm{~ns}$ duration. We obtained a maximum cavity-dumped pulse energy of $50 \mu \mathrm{J}$ at $100 \mathrm{~Hz}$ pulse repetition rate (Fig. 15). The broad amplified spectrum with



Fig. 15. Cavity-dumped amplified pulse energy and average power as a function of repetition rate for the diode-pumped Yb:glass (Kigre QX/Yb phosphate) chirped pulse amplifier a FWHM of $12 \mathrm{~nm}$ could potentially be compressed to 100 -fs-level pulses but, at the time of the experiment, no suitable compressor was accessible. The extraction efficiency with respect to the stored energy of $\approx 770 \mu \mathrm{J}$ (using (5.2)) was $6.5 \%$.

Previously demonstrated diode-pumped Nd:glass CPA systems generated $56 \mu \mathrm{J}$ pulses with an amplified spectrum of $4 \mathrm{~nm}$ FWHM $[78,79]$, resulting in $450 \mathrm{fs}$ pulse width and $23 \mu \mathrm{J}$ pulse energy after compression. The applied pump power of $4 \mathrm{~W}$ was more than 3 times higher than in our Yb:glass amplifier, however, it resulted just in approximately the same extracted pulse energy. Together with the 3 times broader amplified pulse spectrum we conclude that $\mathrm{Yb}$ :glass amplifiers are superior for high peak power generation compared to amplifiers based on Nd-doped glass. As discussed above, this is at the expense of a lower pulse repetition rate.

In the future, higher fs pulse energies should be possible by utilizing laser diodes with higher power. High brightness is crucial for achieving high extraction efficiency. The recently demonstrated high-brightness diodes with $10 \mathrm{~W}$ output $[65,66]$ are particularly interesting for such amplifiers. The good efficiency of the $\mathrm{Yb}^{3+}$ ion helps reduce the thermal problems despite of the low thermal conductivity of the glasses needed as host materials for fs pulse generation.

\section{Conclusion}

We have theoretically shown and experimentally demonstrated the advantages of Yb-doped laser media for short pulse generation. Compared to their Nd-doped counterparts, these media have a high intrinsic efficiency (small quantum defect and absence of parasitic processes), reducing detrimental thermal effects and thus allowing for higher average powers and pulse energies. To realize the potential for high power efficiency, pump sources with high brightness are required as a result of the quasi-three-level nature. Efficient operation with high-brightness laser diodes has been demonstrated, although the low brightness of currently available high-power pump diodes limits the obtainable efficiency. Nevertheless, 1-ps pulses with $1.5 \mathrm{~W}$ of average power from a passively modelocked Yb:YAG laser were demonstrated, and improvements in the brightness of the pump diodes should soon allow for more efficient laser operation. Particularly the peak power from such lasers can be expected to exceed the values obtainable from $\mathrm{Nd}$-doped lasers in the near future.

Still shorter pulses, with a duration as short as $60 \mathrm{fs}$, were generated with diode-pumped Yb:glass lasers. Although this pulse duration is not shorter than obtained so far with $\mathrm{Nd}$ :glass, a much larger tuning range of up to $52 \mathrm{~nm}$ was demonstrated.

The low cross sections of Yb-doped media cause an increased tendency for Q-switching instabilities in passively modelocked lasers, particularly when low-brightness pump sources are used. Various countermeasures have been used to obtain stable cw modelocking in most cases, although the currently available high-power pump diodes do not allow for the suppression of Q-switching instabilities in high-power passively modelocked Yb:glass lasers. Further development of 
power and brightness of diode lasers is expected to make possible Yb-doped fs oscillators with significantly higher average powers compared to $\mathrm{Nd}$ :glass lasers which are limited in power by the stronger thermal effects.

In regenerative amplifiers, Yb-doped media allow higher energies to be stored, while a good extraction efficiency is more sensitive to the cavity loss and also requires a pump source with high brightness. The main attraction of $\mathrm{Yb}: \mathrm{YAG}$ (compared to Nd-doped crystals) is the much larger amplification bandwidth, leading to 2.3-ps short pulses with $180 \mu \mathrm{J}$ energy and $80 \mathrm{MW}$ peak power. Chirped pulse amplification with Yb:glass was also demonstrated and should lead to energetic fs pulses when a suitable compressor is used.

Acknowledgements. The authors thank L.R. Brovelli and C. Harder from Uniphase Laser Enterprise for the high-brightness laser diodes used in the Yb:glass experiments and M. Myers from Kigre Inc. for providing some of the used Yb-doped glass materials. This work was supported by a European grant in Biomed 2 .

\section{References}

1. L.D. DeLoach, S.A. Payne, L.L. Chase, L.K. Smith, W.L. Kway, W.F. Krupke: IEEE J. Quantum Electron. QE-29, 1179 (1993)

2. X. Zou, H. Toratani: Phys. Rev. B 52, 15889 (1995)

3. T.Y. Fan: IEEE J. Quantum Electron. QE-29, 1457 (1993)

4. H.W. Etzel, H.W. Gandy, R.J. Ginther: Appl. Opt. 1, 534 (1962)

5. A.R. Reinberg, L.A. Riseberg, R.M. Brown, R.W. Wacker, W.C. Holton: Appl. Phys. Lett. 19, 11 (1971)

6. P. Lacovara, H.K. Choi, C.A. Wang, R.L. Aggarwal, T.Y. Fan: Opt. Lett. 16, 1089 (1991)

7. A. Giesen, H. Hügel, A. Voss, K. Wittig, U. Brauch, H. Opower: Appl. Phys. B 58, 363 (1994)

8. M. Karszewski, U. Brauch, K. Contag, S. Erhard, A. Giesen, I. Johannsen, C. Stewen, A. Voss, W.R. Bosenberg, M.M. Fejers (Eds.): Adv. Solid State Lasers (ASSL) 1998, p. 296

9. S.A. Payne, L.K. Smith, L.D. DeLoach, W.L. Kway, J.B. Tassano, W.F. Krupke: IEEE J. Quantum Electron. QE-30, 170 (1994)

10. C.D. Marshall, L.K. Smith, R.J. Beach, M.A. Emanuel, K.I. Schaffers, J. Skidmore, S.A. Payne, B.H.T. Chai: IEEE J. Quantum Electron. QE32, 650 (1996)

11. T. Danger, E. Mix, E. Heumann, G. Huber, D. Ehrt, W. Seeber: Adv. Solid State Lasers 1996, p. 23

12. U. Griebner, R. Koch, H. Schönnagel, S. Jiang, M.J. Myers, D. Rhonehouse, S.J. Hamlin, W.A. Clarkson, D.C. Hanna, S.A. Payne, C.R. Pollocks (Eds.): Adv. Solid State Lasers 1996, p. 26

13. R. Koch, W.A. Clarkson, D.C. Hanna, S. Jiang, M.J. Myers, D. Rhonehouse, S.J. Hamlin, U. Griebner, H. Schönnagel: Opt. Commun. 134, 175 (1997)

14. C. Hönninger, F. Morier-Genoud, M. Moser, U. Keller, L.R. Brovelli, C. Harder: Opt. Lett. 23, 126 (1998)

15. D.C. Hanna, R.M. Percival, I.R. Perry, R.G. Smart, P.J. Suni, J.E. Townsend, A.C. Tropper: Electron. Lett. 24, 1111 (1988)

16. H.M. Pask, R.J. Carman, D.C. Hanna, A.C. Tropper, C.J. Mackechnie, P.R. Barber, J.M. Dawes: IEEE J. Sel. Top. Quantum Electron. 1, 2 (1995)

17. R. Paschotta, J. Nilsson, A.C. Tropper, D.C. Hanna: IEEE J. Quantum Electron. QE-33, 1049 (1997)

18. M.H. Muendel, B. Engstrom, D. Kea, B. Laliberte, R. Minns, R. Robinson, B. Rockney, Y. Zhang, R. Collins, P. Gavrilovic, A. Rowley: CLEO 1997, paper CPD30

19. V. Cautaerts, D.J. Richardson, R. Paschotta, D.C. Hanna: Opt. Lett. 22, 316 (1997)

20. C. Hönninger, G. Zhang, U. Keller, A. Giesen: Opt. Lett. 20, 2402 (1995)

21. C. Spielmann, F. Krausz, T. Brabec, E. Wintner, A.J. Schmidt: IEEE J. Quantum Electron. QE-27, 1207 (1991)

22. U. Keller, T.H. Chiu, J.F. Ferguson: Opt. Lett. 18, 1077 (1993)

23. U. Keller, K.J. Weingarten, F.X. Kärtner, D. Kopf, B. Braun, I.D. Jung, R. Fluck, C. Hönninger, N. Matuschek, J. Aus der Au: IEEE J. Sel. Top. Quantum Electron. 2, 435 (1996)
24. U. Keller: In Chapter 4 in Nonlinear Optics in Semiconductors, Vol. 59, ed. by E. Garmire, A. Kost (Academic Press, Boston 1999), p. 211

25. H.A. Haus: IEEE J. Quantum Electron. QE-12, 169 (1976)

26. F.X. Kärtner, L.R. Brovelli, D. Kopf, M. Kamp, I. Calasso, U. Keller: Opt. Eng. 34, 2024 (1995)

27. C. Hönninger, R. Paschotta, F. Morier-Genoud, M. Moser, U. Keller: J. Opt. Soc. Am. B 16, 46 (1999)

28. V. Petrov, U. Griebner, D. Ehrt, W. Seeber: Opt. Lett. 22, 408 (1997)

29. S.R. Henion, P.A. Schulz: CLEO 1992, paper CThQ2 p. 540

30. X. Long, Y.P. Tong, P.M.W. French, J.R. Taylor: Opt. Commun. 141, 162 (1997)

31. C. Hönninger, I. Johannsen, M. Moser, G. Zhang, A. Giesen, U. Keller: Appl. Phys. B 65, 423 (1997)

32. D.R. Walker, C.J. Flood, H.M. v. Driel, U.J. Greiner, H.H. Klingenberg: Appl. Phys. Lett. 65, 1992 (1994)

33. F.H. Loesel, J.P. Fischer, M.H. Götz, C. Horvarth, T. Juhasz, F. Noack, N. Suhm, J.F. Bille: Appl. Phys. B 66, 121 (1998)

34. S. Nolte, C. Momma, H. Jacobs, A. Tünnermann, B.N. Chichkov, B. Wellegehausen, H. Welling: J. Opt. Soc. Am. B 14, 2716 (1997)

35. T.Y. Fan, R.L. Byer: IEEE J. Quantum Electron. QE-23, 605 (1987)

36. W.P. Risk: J. Opt. Soc. Am. B 5, 1412 (1988)

37. W.F. Krupke, L.L. Chase: Opt. Quantum Electron. 22, S1 (1990)

38. P. Peuser, N.P. Schmitt: Diodengepumpte Festkörperlaser (Springer, Berlin, Heidelberg 1994)

39. H.A. Haus, J.G. Fujimoto, E.P. Ippen: J. Opt. Soc. Am. B 8, 2068 (1991)

40. F.X. Kärtner, J. A. d. Au, U. Keller: IEEE J. Sel. Top. Quantum Electron. 4, 159 (1998)

41. U. Keller, D.A.B. Miller, G.D. Boyd, T.H. Chiu, J.F. Ferguson, M.T. Asom: Opt. Lett. 17, 505 (1992)

42. L.R. Brovelli, U. Keller, T.H. Chiu: J. Opt. Soc. Am. B 12, 311 (1995)

43. L.R. Brovelli, I.D. Jung, D. Kopf, M. Kamp, M. Moser, F.X. Kärtner, U. Keller: Electron. Lett. 31, 287 (1995)

44. I.D. Jung, L.R. Brovelli, M. Kamp, U. Keller, M. Moser: Opt. Lett. 20, 1559 (1995)

45. S. Tsuda, W.H. Knox, E.A. d. Souza, W.Y. Jan, J.E. Cunningham: Opt. Lett. 20, 1406 (1995)

46. H.A. Haus: J. Appl. Phys. 46, 3049 (1975)

47. F.X. Kärtner, U. Keller: Opt. Lett. 20, 16 (1995)

48. F.X. Kärtner, I.D. Jung, U. Keller: IEEE J. Sel. Top. Quantum Electron. 2, 540 (1996)

49. H.A. Haus, J.D. Moores, L.E. Nelson: Opt. Lett. 18, 51 (1993)

50. P.F. Curley, C. Spielmann, T. Brabec, F. Krausz, E. Wintner, A.J. Schmidt: Opt. Lett. 18, 54 (1993)

51. T. Kellner, F. Heine, G. Huber, C. Hönninger, B. Braun, F. MorierGenoud, U. Keller: J. Opt. Soc. Am. B 15, 1663 (1998)

52. W. Koechner: Solid-State Laser Engineering (Springer, Berlin, Heidelberg 1992)

53. J. Aus der Au, D. Kopf, F. Morier-Genoud, M. Moser, U. Keller: Opt. Lett. 22, 307 (1997)

54. R.L. Fork, O.E. Martinez, J.P. Gordon: Opt. Lett. 9, 150 (1984)

55. D.E. Spence, P.N. Kean, W. Sibbett: Opt. Lett. 16, 42 (1991)

56. D.K. Negus, L. Spinelli, N. Goldblatt, G. Feugnet: In Advanced SolidState Lasers, Vol. 10 ed. by G. Dubé, L. Chase (Optical Society of America, Washington DC 1991), p. 120

57. U. Keller, G.W. 'tHooft, W.H. Knox, J.E. Cunningham: Opt. Lett. 16, $1022(1991)$

58. F. Salin, J. Squier, M. Piché: Opt. Lett. 16, 1674 (1991)

59. W.A. Clarkson, A.B. Neilson, D.C. Hanna: Conference on Lasers and Electro-Optics 1994, paper CThL2

60. W.A. Clarkson, D.C. Hanna: Opt. Lett. 21, 375 (1996)

61. F. Krausz, J. Zehetner, T. Brabec, E. Wintner: Opt. Lett. 16, 1496 (1991)

62. J. Zehetner, C. Spielmann, F. Krausz, E. Wintner: In Advanced SolidState Lasers, Vol. 13 of OSA Proceedings Series, ed. by L.L. Chase, A.A. Pinto (Optical Society of America, Washington DC 1992), p. 215

63. D. Kopf, K.J. Weingarten, G. Zhang, M. Moser, M.A. Emanuel, R.J. Beach, J.A. Skidmore, U. Keller: Appl. Phys. B 65, 235 (1997)

64. J. Aus der Au, F.H. Loesel, F. Morier-Genoud, M. Moser, U. Keller: Opt. Lett. 23, 271 (1998)

65. A. Al-Muhanna, L.J. Mawst, D. Botez, D.Z. Garbuzov, R.U. Martinelli, J.C. Connolly: Appl. Phys. Lett. 73, 1182 (1998)

66. X. He, S. Srinivasan, S. Wilson, C. Mitchell, R. Patel: Electron. Lett. 34, 2126 (1998) 
67. W.H. Lowdermilk, J.E. Murray: J. Appl. Phys. 51, 2436 (1980)

68. A.E. Siegman: Lasers (University Science Books, Mill Valley, CA 1986)

69. J.S. Coe, P. Maine, P. Bado: J. Opt. Soc. Am. B 5, 2560 (1988)

70. M. Gifford, K.J. Weingarten: Opt. Lett. 17, 1788 (1992)

71. L. Turi, T. Juhasz: Opt. Lett. 20, 154 (1995)

72. M.D. Selker, R.S. Afzal, J.L. Dallas, A.W. Wu: Opt. Lett. 19, 551 (1994)

73. K. Naito, M. Ohmi, K. Ishikawa, M. Akatsuka, M. Yamanaka, M. Nakatsuka, S. Nakai, T. Sato: Appl. Phys. Lett. 64, 1186 (1994)
74. D. Strickland, G. Mourou: Opt. Commun. 56, 219 (1985)

75. D.T. Walton, J. Nees, G. Mourou: Opt. Lett. 21, 1061 (1996)

76. S. Biswal, J. Itatani, J. Nees, G.A. Mourou: IEEE J. Sel. Top. Quantum Electron. 4, 421 (1998)

77. C. Hönninger, S. Biswal, A. Braun, C. Horvath, J. Nees, U. Keller, G. Mourou: CLEO 1998, paper CThJ4

78. A. Braun, X. Liu, G. Mourou, D. Kopf, U. Keller: Appl. Opt. 36, 4163 (1997)

79. C. Horvath, A. Braun, H. Liu, T. Juhasz, G. Mourou: Opt. Lett. 22, 1790 (1997) 